\title{
X-RAYS IN THE ORION NEBULA CLUSTER: CONSTRAINTS ON THE ORIGINS OF MAGNETIC ACTIVITY IN PRE-MAIN-SEQUENCE STARS
}

\author{
Eric D. Feigelson, ${ }^{1}$ James A. Gaffney III, ${ }^{1}$ Gordon Garmire, ${ }^{1}$ Lynne A. Hillenbrand,${ }^{2}$ and Leisa Townsley ${ }^{1}$ \\ Received 2002 July 9; accepted 2002 October 29
}

\begin{abstract}
A recent observation of the Orion Nebula Cluster with the ACIS instrument on board the Chandra X-Ray Observatory detected 1075 sources, 525 of which are pre-main-sequence (PMS) stars with measured bulk properties such as bolometric luminosities, masses, ages, and disk indicators. Nearly half of these stars have photometrically measured rotational periods. This provides a uniquely large and well-defined sample to study the dependence of magnetic activity on bulk properties for stars descending the Hayashi tracks. The following results are obtained: (1) X-ray luminosities $L_{t}$ in the $0.5-8 \mathrm{keV}$ band are strongly correlated with bolometric luminosity, with average ratio $\log L_{t} / L_{\mathrm{bol}}=-3.8$ for stars with masses $0.7<M<2 M_{\odot}$, an order of magnitude below the main-sequence saturation level; (2) the X-ray emission drops rapidly below this level in some stars with $2<M<3 M_{\odot}$; (3) the presence or absence of infrared circumstellar disks has no apparent relation to X-ray levels; and (4) X-ray luminosities exhibit a slight rise as rotational periods increase from 0.4 to 20 days. This last finding stands in dramatic contrast to the strong decline of X-ray emission with increasing period seen in main-sequence stars. The absence of a strong X-ray/rotation relationship in PMS stars, and particularly the high X-ray values seen in some very slowly rotating stars, is a clear indication that the mechanisms of magnetic field generation differ from those operating in main-sequence stars. The most promising possibility is a turbulent dynamo distributed throughout the deep convection zone, but other models, such as $\alpha-\Omega$ dynamo with "supersaturation" or relic core fields, are not immediately excluded. The drop in magnetic activity in intermediate-mass stars may reflect the presence of a significant radiative core. The evidence does not support X-ray production in large-scale star-disk magnetic fields in T Tauri stars.
\end{abstract}

Subject headings: open clusters and associations: individual (Orion Nebula Cluster) — stars: activity stars: magnetic fields — stars: pre-main-sequence — stars: rotation — X-rays: stars

On-line material: machine-readable table

\section{INTRODUCTION}

The astrophysical origin of the surface magnetic activity of solar-type main-sequence stars has been established with some confidence (Schrijver \& Zwaan 2000). Magnetic fields are generated by differential rotation at the interface (tachocline) between the radiative and convective zones and rise through the convection zone to the surface where they produce active regions, violent flares, coronal heating, and other effects. Oscillations in this dynamo account for the 22 yr solar cycle. In other main-sequence stars, the principal evidence for such a dynamo is the ubiquitous relationship between magnetic activity indicators and surface rotation: more rapidly rotating stars exhibit higher levels of activity until, for some indicators, a saturation of the process is reached.

It is not clear, however, whether this model applies to late-type stars with substantially different internal structure from the Sun's, such as pre-main-sequence (PMS) T Tauri stars, post-main-sequence giants, and low-mass M dwarfs. Such stars may have tachoclines buried deep in the interior or may be fully convective without any tachocline. Yet both $\mathrm{T}$ Tauri stars and $\mathrm{dM}$ main-sequence stars exhibit large active regions and strong flaring, indicating that magnetic field generation is operative. Various suggestions have been

\footnotetext{
${ }^{1}$ Department of Astronomy and Astrophysics, 525 Davey Laboratory, Pennsylvania State University, University Park, PA 16802.

${ }^{2}$ Department of Astronomy, MS 105-24, California Institute of Technology, Pasadena, CA 91125.
}

made to account for this, such as a turbulent dynamo process distributed throughout the convective zone, but with little empirical support. Notably, an activity-rotation relationship is sometimes but not always evident in these stars. But the samples for study have generally been small and the empirical results often discrepant.

It has proved difficult to study the origins of magnetic activity in PMS stars using traditional optical and ultraviolet indicators because of obscuration and confusion arising from gas infall and ejections. Elevated levels of X-ray emission, in contrast, are ubiquitous in PMS stars and are relatively unaffected by such problems (see review by Feigelson \& Montmerle 1999). However, despite considerable effort with the Einstein and ROSAT missions, the observational basis for understanding the elevated levels of PMS magnetic activity is still murky. Some studies show an $\mathrm{X}$-ray/rotation correlation, while others do not, and other confounding correlations with bulk properties are present (§ 2.1.3). The theoretical issues are also more complex than with main-sequence stars $(\S 2.2)$.

The Chandra ACIS study of the Orion Nebula Cluster (ONC), which illuminates the M42 blister $\mathrm{H}$ II region on a near edge of the Orion molecular cloud, provides a unique opportunity to study these issues. Here, a single image reveals $\sim 1000 \mathrm{X}$-ray-emitting PMS stars that span the entire initial mass function and a good portion of the PMS evolutionary tracks. The ONC has been the subject of intense optical and infrared study, so that nearly 1000 of its members have been placed on the Hertzsprung-Russell (HR) diagram and over 400 have photometrically measured 
rotation periods. Together, the Chandra and optical results give a great increase in sample size for the study of the origins of PMS magnetic activity compared to previous efforts.

We find no evidence for the X-ray/rotation correlation being strongly present in main-sequence stars among $\mathrm{ONC}$ stars. Various other effects are found that may constrain alternative explanations for PMS magnetic activity. The most promising interpretation, in our view, is that the magnetic fields are produced by a distributed dynamo within the deep convective zone. Further development of theoretical models is needed in light of our observational results.

The paper begins with a review of the complex empirical and theoretical issues concerning magnetic activity and rotation in late-type stars $(\S 2)$. The Chandra ONC data set is presented in $\S 3$, and the effects of various stellar properties on the X-ray emission are explored in $\S 4$. X-ray/ rotation relations are presented in $\S 5$, followed by discussion in $\S 6$ and conclusions in $\S 7$. This is the fourth paper in a series on the Chandra observation of the ONC using the ACIS-I detector: Garmire et al. (2000) introduced the field and discussed stars in the $\mathrm{BN} / \mathrm{KL}$ region; Feigelson et al. (2002a, hereafter F02a) give comprehensive tables of the 1075 sources and discuss X-ray emission as a function of mass; and Feigelson, Garmire, \& Pravdo (2002b, hereafter F02b) discuss flaring in pre-main-sequence analogs of the early Sun and their implications for the early solar system.

\section{STELLAR X-RAYS AND DYNAMOS}

We review here past observational $(\S 2.1)$ and theoretical (§ 2.2) studies which provide the foundation for the present study. We find that the situation for main-sequence $\mathrm{F}-\mathrm{K}$ stars is reasonably clear: rotation appears to be the principal observable correlate to X-ray luminosity and, through the Rossby number, rotation can be linked to an $\alpha$ - $\Omega$-type dynamo that successfully explains many features of solar and stellar activity. The Rossby number $\mathrm{Ro}=P / \tau_{c}$, the ratio of the rotational period $P$ to the convective overturn time $\tau_{c}$ near the base of the stellar convection zone, is a measure of the growth rate of the field in many dynamo theories. Rossby numbers account for mass-dependent structural differences in stellar interiors and are quite stable to reasonable variations in assumptions concerning the physics of the convection zone (Montesinos et al. 2001).

The situation is more confused for giants and dM stars, where only weak activity/rotation relationships are seen. It is not clear whether magnetic fields in the these stars with deep convective zones arise from a modified $\alpha$ - $\Omega$ dynamo or a distributed turbulent dynamo. For PMS stars, the interpretation is even more uncertain: several dynamo concepts compete with the possibility that the magnetic fields are inherited from the gravitational collapse or arise from stardisk interactions.

\subsection{Relationship between Stellar X-Rays and Rotation}

\subsubsection{Solar-Type Main-Sequence Stars}

The surface magnetic activity of solar-type stars arises from the emergence and reconnection of fields generated in the stellar interior (see Schrijver \& Zwaan 2000 for a thorough review). In the X-ray band, this consists of a slowly varying soft $\mathrm{X}$-ray corona and hard emission from violent magnetic reconnection during flares. The first X-ray surveys of late-type stars with the Einstein Observatory revealed a strong X-ray/rotation correlation of the form $L_{s}=$ $10^{27}(v \sin i)^{2} \operatorname{ergs~s}{ }^{-1}$, where $L_{s}$ is measured in the soft 0.5 $2.5 \mathrm{keV}$ band and $v \sin i$ is the projected rotation speed in $\mathrm{km} \mathrm{s}^{-1}$ (Pallavicini et al. 1981). The X-ray/rotation connection for main-sequence stars was repeatedly confirmed in many Einstein and ROSAT studies of both field and open cluster stars.

For later comparison with pre-main-sequence Orion stars, Figure 1 shows two results from these studies. Figure $1 a$ shows a sample of nearby $\simeq 1 M_{\odot}$ field solar analogs, most with ages between 0.3 and several Gyr. The soft X-ray emission closely follows the relation $\log L_{S}=31.1-$ $2.64 \log P \operatorname{ergs~s}{ }^{-1}$, where $P$ is the period in days (Güdel, Guinan, \& Skinner 1997; Gaidos 1998). Figure $1 b$ shows the relation between $\mathrm{X}$-ray emissivity and Rossby number from many ROSAT studies of cluster and field stars (Randich 2000, kindly updated by S. Randich). The lines indicate three regimes (Randich et al. 1996):

1. For slowly rotating stars, X-ray emission is approximately linearly dependent on Rossby number as $\log L_{s} / L_{\text {bol }}=-5.0-2.1 \log$ Ro.

2. Below $\log \mathrm{Ro} \simeq-0.8$, main-sequence stars exhibit a "saturated" X-ray level of $\log L_{s} / L_{\mathrm{bol}}=-3.0$. Saturation is well established for several tracers of magnetic activity in several classes of magnetically active stars (Vilhu \& Walter 1987; Fleming, Schmitt, \& Giampapa 1995; Krishnamurthi et al. 1998). Considered together, all manifestations of surface magnetic fields should not exceed $\sim 1 \% L_{\text {bol }}$, a general limit on the mechanical power in convection (Mullan 1984). But other saturation processes may also be involved, such as a limit of field generation capacity of the underlying dynamo, complete coverage of the surface by strong fields (unity filling factor of photometric starspots), or centrifugal forces on large magnetic loops in rapidly rotating coronae (Randich 1998; Jardine \& Unruh 1999).

3. The most rapidly rotating stars with $P<0.5$ days lie in a "supersaturated" regime where X-ray emission drops several-fold below the saturation limit. Cluster "ultrafast rotators" with $v \sin i \simeq 100-200 \mathrm{~km} \mathrm{~s}^{-1}$, rotationally coupled W UMa binary stars, and some dM stars exhibit supersaturation. Again, the cause of the diminution of activity is uncertain: perhaps magnetic flux is concentrated toward the poles, centrifugal forces limit the coronal extent, or coronal temperatures lie out of the narrow ROSAT passband in these rapidly rotating stars (Randich 1998; James et al. 2000; Stepień, Schmitt, \& Voges 2001; Mullan \& MacDonald 2001).

Despite these interpretational difficulties and some discrepancies between different samples, the overall agreement over 3.5 orders of magnitude of X-ray luminosity seen in Figure $1 b$ is probably the clearest empirical indicator of the underlying relationship between magnetic activity and stellar angular momentum (Krishnamurthi et al. 1998). In particular, the dependence of $L_{S} / L_{\text {bol }}$ on mass appears to be relatively weak in main-sequence stars, in contrast to the findings we report here for PMS stars (§ 4.3).

\subsection{2. $d M$ and Giant Stars}

The $\alpha-\Omega$ dynamo model is less convincing for stars with very deep convective zones, such as M-type dwarfs and post-main-sequence giants; for these stars, the activityrotation relation is confusing and poorly understood. This 


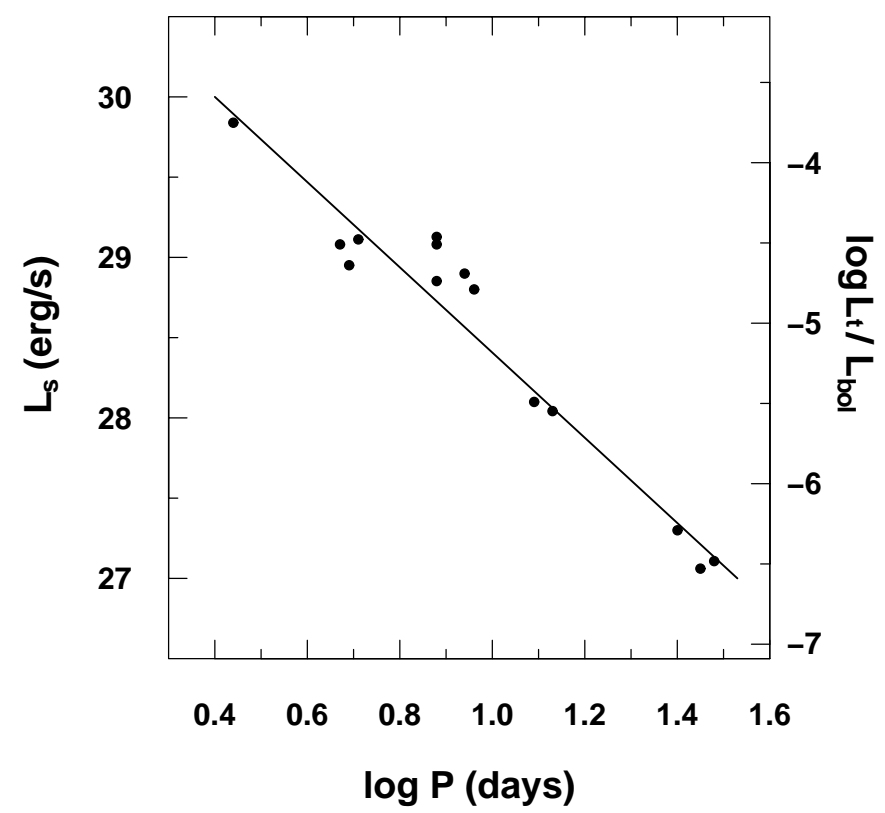

FIG. $1 a$

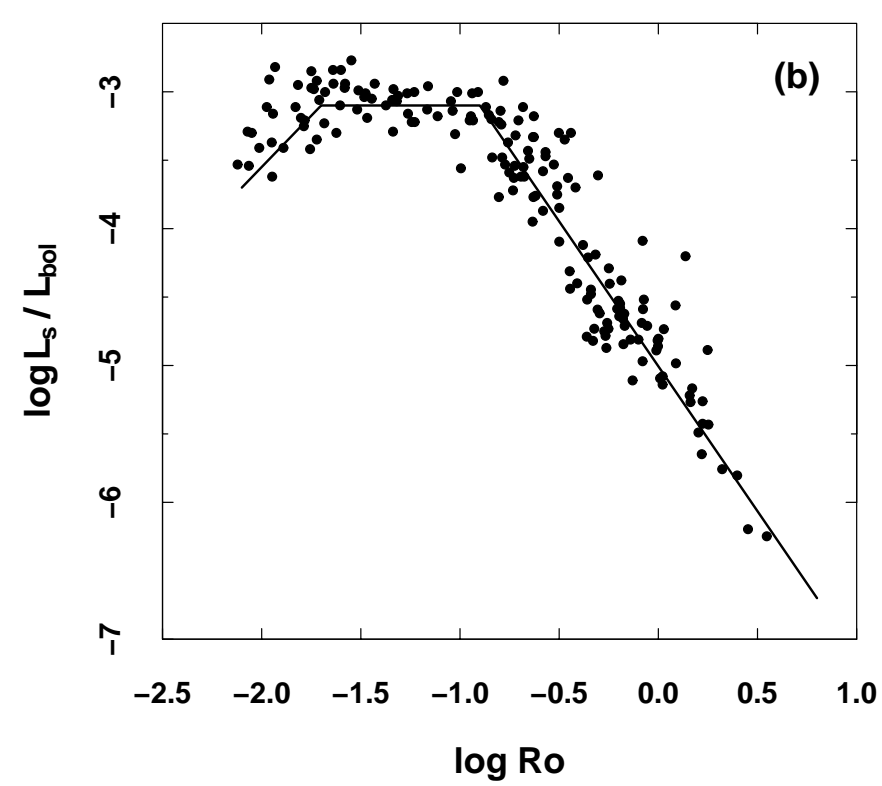

FIG. $1 b$

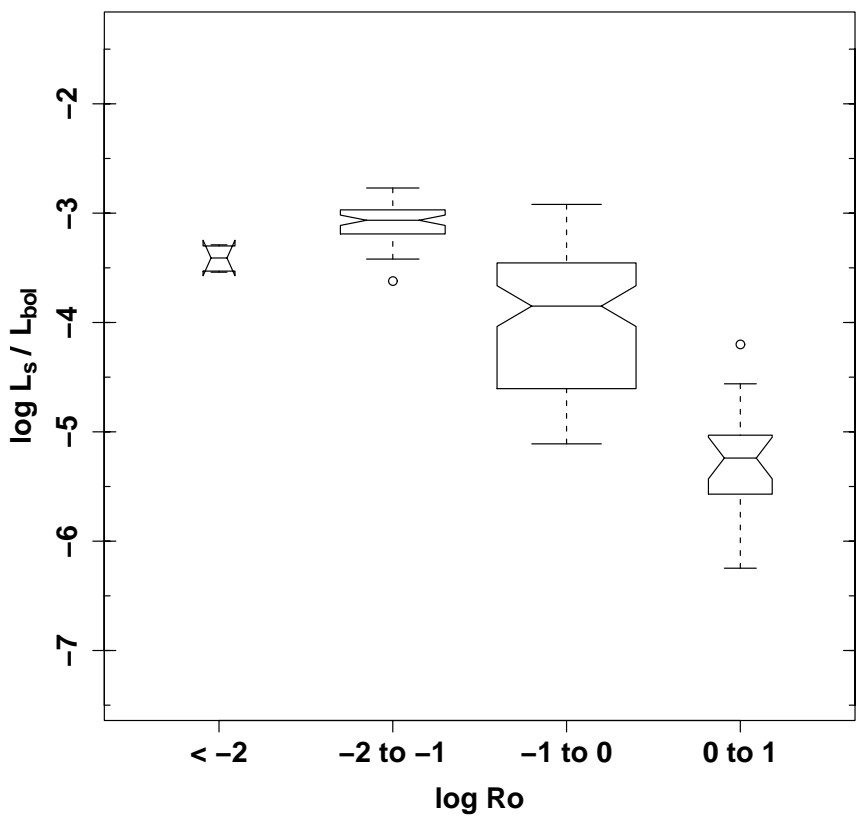

FIG. $1 c$

FIG. 1.-Relationships between soft X-ray emission and rotation in main-sequence stars from ROSAT studies: $(a) L_{s}$ vs. rotation period $P$ for $1 M_{\odot}$ solar analogs; $(b)$ scatter plot $L_{s} / L_{\mathrm{bol}}$ vs. Rossby number for open clusters and field stars; and $(c)$ box plot of $L_{s} / L_{\mathrm{bol}}$ vs. Rossby number for open clusters and field stars. The lines here show the X-ray/rotation correlation (right), saturated (middle), and supersaturated (left) regimes. See $\S 2.1 .1$ for references and $\S 3.2$ for a description of the box plot.

departure from solar-type main-sequence stars is particularly relevant to PMS stars, which are fully convective at the birthline and (except for very low mass stars) develop radiative cores as they descend the Hayashi tracks.

Standard interiors models indicate that the convective zone thickens as mass decreases on the main sequence and the stars become fully convective below mass $0.3-0.4 M_{\odot}$ (M3-M4). Yet, no change in either the distribution of rotational velocities or the activity/rotation relation is seen around this spectral type (Delfosse et al. 1998). This may be explained by deficiencies in standard interior models that neglect to consider how magnetic fields can suppress the onset of complete convection down to $\simeq 0.1 M_{\odot}$ (Mullan \& MacDonald 2001). There may be a subset of M dwarfs in which the surface activity does not depend on rotation; these may be cases in which the fields are generated throughout the convection zone. The rotational evolution of $\mathrm{dM}$ stars may be simpler than for higher mass stars, as there is less opportunity for internal redistribution of angular momentum (Sills, Pinsonneault, \& Terndrup 2000).

Considerable study has been made of magnetic activity of giants with masses $1<M<3 M_{\odot}$ and bolometric luminosities $3<L_{\text {bol }}<100 L_{\odot}$ lying at the base of the red giant branch after crossing the Hertzsprung gap, occupying the 
same region of the HR diagram as $<1$ Myr T Tauri stars. Their interiors range from nearly fully radiative $G$ giants to $\mathrm{K}$ giants with an outer convective zone occupying $90 \%$ of the stellar radius. The strongest effect among these stars is the "coronal dividing line": giants with spectral types hotter than about K1 typically exhibit $\log L_{s} \sim 28-30$ ergs s$^{-1}$ $\left(\log L_{s} / L_{\text {bol }} \sim-7\right.$ to -5$)$, while cooler giants are usually X-ray inactive, sometimes with $\log L_{s} / L_{\text {bol }} \leq-10$ (e.g., Ayres et al. 1981; Huensch et al. 1996; Gondoin 1999).

While a rough link between X-ray luminosity and rotation is present because both are low for the cooler giants, the X-ray/rotation diagram for the hotter giants shows mostly scatter, up to 3 orders of magnitude in $L_{s}$ for a given rotational velocity (Gondoin 1999; Pizzolato, Maggio, \& Sciortino 2000). Several stars are known with slow rotation $\left(v \sin i \simeq 1-3 \quad \mathrm{~km} \mathrm{~s}^{-1}\right)$ and high $\mathrm{X}$-ray luminosities $\left(\log L_{s} \sim 29-30.5 \mathrm{ergs} \mathrm{s}^{-1}\right)$. A weak X-ray/rotation correlation may be present for the lower mass $\left(1.0<M<1.5 M_{\odot}\right)$ giants, but an anticorrelation between $L_{s}$ and $v \sin i$ may be present among higher mass $\left(1.5<M<3.0 M_{\odot}\right)$ giants. These authors suggest that the strength of the dynamo in these more massive giants is regulated more by internal differential rotation than by the rotation itself. Computations indicate that turbulence-induced differ-

ential rotation arises as the convective envelope thickens (Kitchatinov \& Rüdiger 1999). However, it is possible that the coronal dividing line arises from differences in magnetic field configurations at the stellar surface rather than differences in dynamo processes (Rosner et al. 1995). A valuable but inconclusive discussion on issues concerning magnetic activity in red giants appears in Strassmeier et al. (1998).

\subsubsection{Pre-Main-Sequence Stars}

High levels of X-ray emission are ubiquitous among PMS stars, with the X-ray luminosity function extending from less than $10^{28}$ to $10^{31} \mathrm{ergs} \mathrm{s}^{-1}$ (see review by Feigelson \& Montmerle 1999). This is far above typical main-sequence levels of $10^{26}-10^{29} \mathrm{ergs} \mathrm{s}^{-1}$ but, because their surface areas are greater, their surface fluxes are typically an order of magnitude below main-sequence saturation levels. The emission is characterized by high temperatures $(k T \simeq 2 \mathrm{keV}$ is typical but 5 to $>10 \mathrm{keV}$ values are not uncommon; F02a), too hot to be produced by an accretion shock. The $\mathrm{X}$-ray emission is usually strongly variable; for example, the Chandra data set studied here indicates that solar mass ONC stars exhibit flares with $L_{t}$ (peak) $\geq 10^{29} \mathrm{ergs} \mathrm{s}^{-1}$ every few days (F02b). The emission is thus dominated by flares rather than by a soft-spectrum, quiescent corona. The geometry of the reconnecting fields responsible for the flares is quite uncertain. Possibilities include field lines rooted in the stellar surface as in older stars, field lines extending from the star to the disk, and fields in a disk corona.

The relationship between activity and rotation for PMS stars is not well established. Although elevated X-ray emission is present during all PMS phases, rotation is more easily measured during the later phases, when the continuum and sometimes broad emission line excesses of the "classical" T Tauri phase have subsided. Most of the measured periods are obtained from photometric time series of rotationally modulated cool starspots on "weak-lined" $\mathrm{T}$ Tauri stars, which are no longer interacting with their circumstellar disks (e.g., Herbst et al. 2002). A handful of bright T Tauri stars also have surface Doppler images (e.g.,
Donati 1999; Granzer et al. 2000) and Zeeman magnetic field measurements (Johns-Krull, Valenti, \& Koresko 1999).

X-ray/rotation studies have concentrated on $\mathrm{T}$ Tauri stars in the Taurus-Auriga complex $(d \simeq 140 \mathrm{pc})$, which are often well studied and not heavily obscured. Promising evidence for a solar-type dynamo emerged from the Einstein Observatory when Bouvier (1990) reported an anticorrelation between $F_{s}=L_{s} / 4 \pi R_{*}^{2}$ and rotation period in a sample of 13 classical and 8 weak-lined T Tauri stars. Their X-ray activity is elevated several-fold above active main-sequence stars with similar rotations. However, the correlation is weaker and the scatter greater when a larger Einstein sample of 50 Taurus-Auriga stars are considered (Damiani \& Micela 1995). Studies of the entire Taurus-Auriga region with the ROSAT All-Sky Survey gave large samples showing apparent correlations between X-ray luminosities and rotational periods and surface velocities (Neuhäuser et al. 1995; Wichmann et al. 2000; Stelzer \& Neuhäuser 2001). These results are discussed with respect to our findings in $\S 5.1$.

The X-ray/rotation relation has also been sought in other nearby star-forming regions. ROSAT studies of the Chamaeleon I cloud and the ONC, for example, show most stars lying below the saturation level without an evident X-ray/ rotation correlation (Feigelson et al. 1993; Gagné, Caillault, \& Stauffer 1995). Two ROSAT samples selected for unusually strong X-ray emission similarly show no X-ray/ rotation correlation, with several stars overluminous in $\mathrm{X}$-rays compared to saturated main-sequence stars (Preibisch 1997; Alcalá et al. 2000).

In summary, a broad correlation with rotational speed is present in some samples, but considerable scatter is present and the relationship may not be the same as seen in mainsequence stars (Fig. 1). Note, however, that previous investigations generally had samples too small to permit study of the rotational effects on X-ray activity independent of other properties such as stellar mass. ${ }^{3}$

\subsection{Theoretical Considerations}

The standard dynamo theory developed for the solar interior and applied to main-sequence and giant stars as outlined above cannot be readily applied to fully convective stars, as it assumes the field is generated and amplified at the interface, or tachocline, between the convective and radiative zones. However, models have been developed in which dynamos operate throughout a convection zone (Durney, De Young, \& Roxburgh 1993). If sufficiently efficient, such a distributed dynamo could not only explain surface magnetic activity, but could have a considerable effect on the bulk stellar properties. For example, a field with $3 \%$ of the energy density of the gas distributed throughout the interior of PMS stars shifts the Hayashi tracks several hundred

\footnotetext{
${ }^{3}$ We do not address here the complex and poorly understood astrophysics of the rotational evolution of PMS stars. Possible stages include: spin-up during the star formation process when accretion from the cloud envelope dominates; spin-down due to magnetic coupling between the star and disk; spin-up due to angular momentum conservation as the star descends the Hayashi track; and spin-down during passage to the main sequence, due either to braking by a magnetic stellar wind or redistribution of angular momentum between the core and envelope (e.g., Bodenheimer 1995; Bouvier, Forestini, \& Allain 1997a; Stassun et al. 1999; Barnes, Sofia, \& Pinsonneault 2001; Tinker, Pinsonneault, \& Terndrup 2002).
} 
degrees toward the red compared to standard tracks in the HR diagram (D’Antona, Ventura, \& Mazzitelli 2000).

\subsection{1. $\alpha-\Omega$ Solar-Type Dynamo}

In a modern dynamo theory for Sun-like stars (e.g., Parker 1993; Charbonneau \& MacGregor 1997; Markiel \& Thomas 1999), a toroidal field is generated by strong differential rotation that arises in the thin overshoot layer or tachocline between the radiative and convective zones (the $\Omega$ effect). These fields are then twisted and transported through the rotating convective zone to the surface (the $\alpha$ effect). With an appropriate choice of $\alpha$, such models explain many characteristics of solar activity, including the $22 \mathrm{yr}$ cycle, the "butterfly diagram" of active region magnetic orientations, and differential rotation in the solar interior inferred from inversion of helioseismological data (e.g., Charbonneau et al. 1999).

For dynamo mechanisms that scale with the Rossby number, the deep convective zones of PMS stars lead to $\tau_{c}$ values an order of magnitude longer than in main-sequence stars, giving smaller Ro values and more magnetic field generation at a given rotational period compared to main-sequence stars. However, the relevance of Ro for PMS magnetic field generation is not clear. For example, Durney \& Robinson (1982) suggest that for a distributed dynamo, the efficiency scales with the depth of the convective region as well as the inverse of the Rossby number.

Two detailed calculations of the convective turnover time $\tau_{c}$, and hence Rossby numbers, for PMS stars have been reported. First, Gilliland (1986) considered nonrotating PMS interior and finds $\tau_{c} \sim 200$ days for fully convective PMS stars at the top of the Hayashi track. In higher mass stars, $\tau_{c}$ drops sharply by several orders of magnitude in $\simeq 1$ (10) $\mathrm{Myr}$ for $M=3 M_{\odot}\left(1 M_{\odot}\right)$ stars. In lower mass $0.5-1$ $M_{\odot}$ stars, $\tau_{c}$ falls only gradually over $10^{7}-10^{8} \mathrm{yr}$. Second, Kim \& Demarque (1996) provide calculations of $\tau_{c}$ using updated OPAL opacities, realistic surface boundary conditions, improved models of diffusion and rotational mixing, and angular momentum loss by a magnetized stellar wind. They treat fully convective Hayashi track stars with masses between 0.5 and $1.2 M_{\odot}$ undergoing solid-body rotation with equatorial surface velocity of $30 \mathrm{~km} \mathrm{~s}^{-1}$ (corresponding to a period $P \simeq 5$ days if $\left.R_{*}=3 R_{\odot}\right)$. Surface rotation is assumed to decay with age as $t^{-1 / 2}$ (which may often not be correct). They find that $\tau_{c}$ rises from around 600 to $\geq 1000$ days over several million years in $0.5-1 M_{\odot}$ stars, after which it drops to shorter timescales. More massive 1.0-1.2 $M_{\odot}$ stars start at $\tau_{c} \simeq 700-400$ days and only show the decline. This implies that dynamo efficiency is constant (for solar-mass) or grows 1-2 orders of magnitude (for subsolar mass) stars during the first $\sim 10 \mathrm{Myr}$, after which it drops by several orders of magnitude over gigayear timescales.

We use $\tau_{c}$ values from Kim \& Demarque (1996) in deriving Ro values for ONC stars below. We caution that the calculations of $\tau_{c}$ by Gilliland (1986) and Kim \& Demarque (1996) differ both in qualitative behavior and quantitatively by factors of 2-5 over the age range of interest, and even the relevance of the Rossby number for magnetic field generation or surface magnetic activity in these stars is uncertain.

\subsubsection{Distributed Dynamos}

A distributed dynamo due to turbulence in the convection zone was first discussed in detail by Durney et al. (1993).
They emphasize that the turbulent velocity field in a convection zone will generate small-scale magnetic fields that can attain energy densities comparable to the kinetic energy density of convective motions. Rotation may enhance the rate of field generation but is not essential to the process. The principal result of adding an $\Omega$ effect from the boundary between a convection zone and a radiative core is to build significant energy densities in large-scale fields, such as those that dominate the solar cycle. They argue that small-scale turbulent fields may coexist with large-scale $\alpha-\Omega$ fields generated in the tachocline, and should dominate the largescale fields in stars with deep convective zones.

Recent calculations have been made of fully convective $T$ Tauri stars rotating nearly as a solid body with differential rotation around $1 \%$, both radially within the convection zone and latitudinally along the surface (Küker \& Rüdiger 1997; Kitchatinov \& Rüdiger 1999; Küker \& Stix 2001). Field amplification occurs throughout the convection zone, and little dependence on bulk rotation is expected. In other models of PMS interiors, magnetic activity is inferred to arise from $\alpha-\alpha$ processes, producing nonaxisymmetric and steady fields, in contrast to $\alpha-\Omega$ fields, which are typically axisymmetric and oscillatory (Moss 1996; Küker \& Rüdiger 1999; Kitchatinov 2001).

Schrijver \& Zwaan (2000, p. 183f) outline a related dynamo concept for stars with deep convective envelopes. At the base of the convective zones, where the Alfvén velocity is low, magnetic fields are subject to little buoyancy and reside in the same region for a long time. They are then wound up and greatly strengthened by differential rotation, giving a strong field layer analogous to the tachocline in solar-type stars from which an $\alpha-\Omega$ dynamo can be sustained.

Mullan \& MacDonald (2001) give a valuable discussion concerning whether a sharp change in X-ray emission is expected in a star (or ensemble of stars) that passes from a core-convection zone structure to a completely convective structure. No clear prediction can be made: turning off an efficient $\alpha$ - $\Omega$ dynamo should reduce the $\mathrm{X}$-ray emission, but the less efficient $\alpha$ - $\alpha$ dynamo may compensate by operating over a larger volume.

Finally, we note that distributed dynamo theories refer to field generation in the stellar interior and do not specify how these fields emerge onto the surface to produce the extremely large starspots and violent X-ray flares observed in PMS stars. A critical issue is whether the surface magnetic saturation level, as measured by $L_{s} / L_{\text {bol }}$, could be substantially lower for a distributed dynamo than a main-sequence $\alpha-\Omega$ dynamo.

\subsubsection{Relic and Core Magnetic Fields}

It is possible that the dominant source of magnetic flux in T Tauri stars are "fossil fields" inherited from the star formation process rather than generated by a dynamo (Mestel 1999). Poloidal magnetic fields of the order of $10^{4} \mathrm{G}$ are roughly expected from compression of interstellar cloud fields (Dudorov et al. 1989; Levy, Ruzmaikin, \& Ruzmaikina 1991). In a fully convective PMS star, this fossil interstellar field should quickly decay as a result of turbulent magnetic diffusivity. However, it is possible that the field may collect into flux ropes that would resist turbulent diffusion until a radiative core develops (Moss 2002). 
PMS magnetic fields might also arise in the radiative core (which forms at $t \simeq 2 \mathrm{Myr}$ for a $1 M_{\odot}$ star) by capturing flux from the convective zone. Such core fields could persist unchanged for billions of years and could coexist with convective zone dynamo-generated fields (Tayler 1987; Moss 1996; Kitchatinov, Jardine, \& Collier Cameron 2001). Relic fields trapped in the larger radiative cores of intermediatemass stars may account for the high surface fields in Am/ Ap stars (Mullan 1973; Stepień 2000). Unlike dynamo-generated fields, relic fields are likely to have a global dipole component and may be nonaxisymmetric (Kitchatinov 2001). A global dipole is needed to produce the large-scale field lines thought to link the T Tauri star to the circumstellar disk at the corotation radius (e.g., Hartmann 1998).

\subsubsection{Disk-related Fields}

$\mathrm{T}$ Tauri stars differ from older late-type stars in that they often have a circumstellar disk. While the disk is thermodynamically cold and neutral, sufficient X-rays and cosmic rays likely penetrate and ionize the disk to freeze in magnetic fields and initiate MHD instabilities and dynamo processes (Glassgold, Feigelson, \& Montmerle 2000). Some forms of magnetic activity, such as the reconnection flares that dominate the X-ray emission, may thus arise in three locations: at the stellar surface, as in other late-type stars; at the corotation interface between large-scale dipolar stellar fields and the inner disk (Shu et al. 1997; Montmerle et al. 2000; Birk et al. 2000); or above the disk in a magnetically active corona (e.g., Levy \& Araki 1989; Romanova et al. 1998; Merloni \& Fabian 2001). There is a wealth of evidence for strong activity at the stellar surface, but the strong fluorescent $6.4 \mathrm{keV}$ iron line seen in two protostars (Koyama et al. 1996; Imanishi, Koyama, \& Tsuboi 2001) may be evidence that X-ray flares occur in close proximity to the disk. This issue of the geometry of reconnecting magnetic field lines in T Tauri systems is discussed in detail by F02b.

\section{THE X-RAY DATA}

\subsection{Observations}

The Orion Nebula Cluster (ONC) is the richest young star cluster within $500 \mathrm{pc}$, with $\simeq 2000$ members concentrated in a $1 \mathrm{pc}\left(8^{\prime}\right)$ radius sphere (O’Dell 2001). The full initial mass function from a $45 M_{\odot}$ O star to dozens of substellar brown dwarfs is present. Over 1500 stars are not deeply embedded and have $V<20 \mathrm{mag} ; \sim 1000$ of these have high-quality photometry and spectroscopy (Hillenbrand 1997 and subsequent updates to the database). This gives locations on the HR diagram from which stellar ages and masses are inferred from theoretical stellar interior models (D'Antona \& Mazzitelli 1997). We ignore here the X-ray population of deeply embedded stars that lies behind the ONC around the OMC 1 cloud cores.

The ONC was observed with the ACIS-I imaging array on board Chandra twice during the inaugural year of the satellite, on 1999 October 12 and 2000 April 1, for $\simeq 12 \mathrm{hr}$ on each occasion. The satellite and instrument are described by Weisskopf et al. (2002). The reader should consult F02a for an atlas of the field, full description of the data-reduction procedures, and properties of the $1075 \mathrm{X}$-ray sources found in the field.

\subsection{Sample and Database}

Of the 1075 ACIS ONC sources, we consider stars with estimated ages and masses (Hillenbrand 1997) and further eliminate stars with $M>3 M_{\odot} .{ }^{4}$ The resulting sample of 525 stars is listed in Table 1. Absorption is not large for most of these stars: $47 \%$ have $A_{V} \leq 1,95 \%$ have $A_{V}<5$, and for $77 \%$ the difference between the observed total band $\left(\log L_{t}\right)$ and absorption-corrected $\left(\log L_{c}\right) \mathrm{X}$-ray luminosities does not exceed 0.3 . The $\log L_{t}$ values in the $0.5-8 \mathrm{keV}$ band thus reflect the true emission with reasonable accuracy. The $\log L_{s}$ luminosities in the soft $0.5-2 \mathrm{keV}$ band will be more seriously affected by absorption, and are provided only to permit comparison with earlier ROSAT soft band results. Note that the main source of scatter in the X-ray luminosi-

\footnotetext{
${ }^{4}$ For intermediate- and high-mass ONC stars with $M>3 M_{\odot}$, it is not clear that the X-rays arise from the optically characterized star rather than from unseen companions (F02a, $\S \S 5.1-5.2$ ). Only one of these omitted stars has a measured rotation period: the B8 star JW 660, with period of 6.15 days and a high X-ray luminosity of $\log L_{t}=31.1 \mathrm{ergs} \mathrm{s}^{-1}(0.5-8 \mathrm{keV}$ band).
}

TABLE 1

X-Ray Properties of Well-characterized ONC Stars

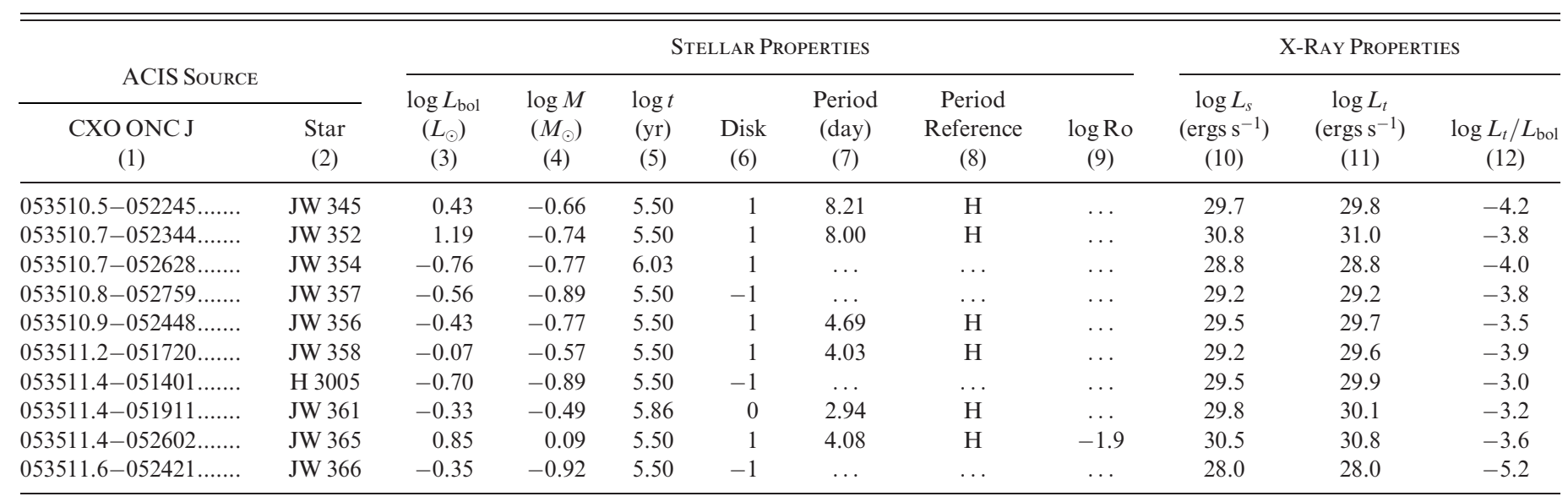

NoTE.-Table 1 is published in its entirety in the electronic edition of the Astrophysical Journal. A portion is shown here for guidance regarding its form and content. 
ties is the intrinsic variability of the sources during the two observations.

Table 1 gives the ACIS-I CXO ONC source name (col. [1]); associated optical star (col. [2], most are designated JW from Jones \& Walker 1988); stellar bolometric luminosity, mass, and age (cols. [3]-[5]); a circumstellar disk indicator (col. [6]); rotational period with reference (cols. [7] and [8]); estimated Rossby number (col. [9]); soft and total band $\mathrm{X}$-ray luminosities (cols. [10] and [11]), and the ratio of total band X-ray to bolometric luminosity (col. [12]). Columns (1)-(5) and (10)-(11) are extracted from Tables 2 and 3 of F02a. As in F02b, we considered stellar ages below $\log t=5.5$ yr to be upper limits because of difficulties in establishing the zero-age point in evolutionary calculations (e.g., Wuchterl \& Klessen 2001). The disk indicator is based on the criteria given by F02b with data from F02a. A plus symbol indicates a near-infrared photometric excess $\Delta(I-K)>0.3$ and/or association with a Herbig-Haro outflow, far-infrared source or imaged proplyd; a minus symbol indicates $\Delta(I-K)<0.3$ and no association of these types; and ellipses indicate insufficient information for classification. The $\Delta(I-K)$ measurements are from Hillenbrand et al. (1998).

The photometric rotational periods are extracted from Table 2 of F02a. The code for rotation period references is: $\mathrm{C}=$ Carpenter, Hillenbrand, \& Skrutskie (2001); $\mathrm{H}=$ Herbst et al. (2000, 2002); and $S=$ Stassun et al. (1999). A few rotation periods have been updated from those given in F02a based on the final results of Herbst et al. (2002), and stars with discrepant reported photometric periods are listed in the Notes to Table 2 of F02a. We do not supplement these with 43 new periods estimated from the projected Doppler surface velocity measured spectroscopically by Rhode, Herbst, \& Mathieu (2001). Periods derived from spectroscopy are inaccurate because of the unknown inclinations of individual stars, and a systematic overestimation compared to photometric periods is present.

Column (9) of Table 1 lists Rossby numbers (Ro) derived from the observed rotation periods and $\tau_{c}$ estimated from Figure 3 of Kim \& Demarque (1996) in the 0.5-1.2 $M_{\odot}$ range $(\S 2.2 .1)$. Because of these restrictions, only 36 values are given.

Columns (10)-(12) give the X-ray luminosities $\log L_{s}$ $\left(\operatorname{ergs~s}^{-1}\right)$ in the soft $0.5-2 \mathrm{keV}$ band, $\log L_{t}$ in the total $0.5-8$ $\mathrm{keV}$ band, and the ratio $\log L_{t} / L_{\mathrm{bol}}$, where $L_{\mathrm{bol}}$ is obtained from Hillenbrand (1997). The $\log L_{s}$ and $\log L_{t}$ values are obtained from Table 3 of F02a; see their $\S \S 2.6-2.9$ for details. $^{5}$

In $\S \S 4-5$, we visualize the data from Table 1 using box plots in addition to two-dimensional scatter plots. Box plots are a simple nonparametric graphical tool for visualizing and comparing univariate distributions widely used in many fields (Tukey 1977; McGill, Tukey, \& Larsen 1978). The

\footnotetext{
${ }^{5}$ We provide $\log L_{s}$ values to facilitate comparison of the Chandra results to earlier ROSAT results. When comparing PMS to main-sequence $\mathrm{X}$-ray emissivities, recall that the $R O S A T$-derived $\log L_{S} / L_{\mathrm{bol}}$ values for main-sequence populations are systematically lower than our $\log L_{t} / L_{\text {bol }}$ value because of our wider bandwidth $\left(0.5-2 \mathrm{keV}\right.$ for $L_{s}$ vs. $0.5-8 \mathrm{keV}$ for $\left.L_{t}\right)$. For typical PMS spectra, $L_{t}$ values are typically a factor of 2 higher than $L_{s}$ values because of this bandwidth effect, and may be higher yet because of interstellar attenuation of $L_{s}$. In particular, we note that the ROSAT-derived main-sequence saturation level $\log L_{s} / L_{\mathrm{bol}}=-3.0$ (Fig. $1 b$ ) is equivalent to about $\log L_{t} / L_{\mathrm{bol}} \simeq-2.7$ for lightly absorbed stars.
}

center of the box indicates the median value and the " hinges" (ends) of the box enclose the 25\% and 75\% quartiles of the data. "Whiskers" (error bars) extend from the box to the largest data value less than 1.5 times the quartile range. Circles show outliers if present; for a Gaussian distribution, about 1 in 100 points will be an outlier. If the "notches" (indented regions around the medians) of two boxes on the same plot do not overlap, then the two population medians are different with greater than $95 \%$ confidence based on an assumption of asymptotic normality of the standard deviation of the medians (i.e., large- $N$ samples). The width of the boxes is scaled to the square root of the number of points included in each box, so that the wider boxes have greater statistical reliability than narrower boxes. The range of each box along the abscissa was chosen by us in an arbitrary manner. The graphics were produced with R (Ihaka \& Gentleman 1996), ${ }^{6}$ a public-domain statistical software package closely related to the commercial S-Plus package.

\subsection{Sample Completeness}

Although Table 1 is by far the largest data set of magnetic activity measurements for PMS stars with measured stellar properties, we must consider systematic biases present in the sample:

1. Our sample is first restricted to 979 ONC stars placed on the HR diagram lying within the ACIS field. This sample is estimated to be $100 \%$ complete for all ONC stars with $M \geq 0.5 M_{\odot}$ with $A_{V} \leq 0$, and for $A_{V}<2.5100 \%$ complete for $M \geq 1 M_{\odot}$ and $50 \%-70 \%$ complete above the substellar limit (Hillenbrand 1997, § 4.3). The main omissions are very low mass $M$ stars and brown dwarfs, which show up in deep K-band studies (Hillenbrand \& Carpenter 2000).

2. Of these 979 stars, our sample is restricted to 525 stars detected with Chandra having ACIS count rates above $0.1-$ 0.4 counts $\mathrm{ks}^{-1}$ in the $0.5-8 \mathrm{keV}$ band, where the higher values are due to reduced sensitivities from the poor pointspread function toward the outer portions of the cluster (F02a, $\S 2.12)$. For most cluster members with typical intrinsic PMS X-ray spectra and low absorptions, this limit corresponds to $\log L_{t}=28.0-28.5 \mathrm{ergs} \mathrm{s}^{-1}$, although some limits reach $\log L_{t}=29.0 \mathrm{ergs} \mathrm{s}^{-1}$. Here also a strong bias in mass is present: $\simeq 90 \%(\mathrm{~F} 02 \mathrm{a}, \oint 5.2)$ of ONC members with $M>1.5 M_{\odot}$ are present, compared to roughly $25 \%$ of PMS brown dwarfs (F02a, $\S 5.6)$.

3. Of these 525 stars, 232 have measured photometric periodicities interpreted as rotationally modulated starspots. By comparing spectroscopically measured $v$ sin $i$ rotational velocities for ONC stars with and without detected photometric starspots, Rhode, Herbst, \& Mathieu (2001) have found that the stars with modulated starspots have the same rotational distribution as the underlying ONC population. Also, the latest study of Herbst et al. (2002), which provides most of the photometric rotation periods used here, extends period measurements down to $M \simeq 0.1 M_{\odot}$. The rotation measurements should thus not contribute any further bias to our sample except below $M \simeq 0.1 M_{\odot}$.

4. Both the optical and X-ray data have arcsecond $(\sim 500$ $\mathrm{AU})$ resolution and thus see the majority of binary and

\footnotetext{
${ }^{6} \mathrm{R}$ software and documentation can be obtained at http://www.r-project.org.
} 
multiple systems as single stars (Mathieu 1994). We assume that both the optical and X-ray light is dominated by a single primary component. This assumption also tends to deemphasize the presence of lower mass stars from our sample.

We conclude that the principal bias in our sample of 525 stars involves stellar mass and associated variables such as bolometric luminosity. A double bias is present: the underlying optical sample is deficient in low-mass stars compared to the underlying cluster, and the X-ray observation is deficient in detecting these stars. A more complete sample would thus have many more objects at low masses with characteristically lower X-ray luminosities. The bias is nearly absent for masses $0.7<M<3 M_{\odot}$. From Table 5 in F02a and Table 1 in F02b, we find that there are only four ${ }^{7}$ undetected stars in this mass range: P1892, with $M=2.6$ $M_{\odot}$; JW 531, with $M=2.5 M_{\odot} ;$ JW 608, with $M=1.8 M_{\odot}$; and JW 62, with $M=1.4 M_{\odot}$. These stars are shown as arrows in some of the figures below.

\subsection{Sources of Uncertainty}

As considerable scatter appears in the correlation plots presented below, it is important to discriminate the degree to which these arise from measurement errors or from true astrophysical variance. The broadband $0.5-8 \mathrm{keV}$ X-ray luminosities $\log L_{t}$ in most cases have rather small $\left(\Delta \log L_{t}= \pm 0.1\right)$ statistical uncertainties, but the intrinsic variability due to X-ray flaring is frequently $\Delta \log L_{t}= \pm 0.3$ during the two $12 \mathrm{hr}$ Chandra observations (F02a, $\S 2.9$ ) and is sometimes considerably greater (F02b). The long-term variability of a star will obviously exceed the variability found during the limited observations available here. We thus expect all samples of PMS stars to exhibit significant scatter in X-ray luminosity, roughly $\Delta \log L_{t}= \pm 0.5$ for the majority of stars, due to statistics and variability.

Uncertainty or systematic errors may also be present in other stellar parameters. The value of $\log L_{\mathrm{bol}}$ is relatively well established, with errors about \pm 0.15 by the photometry and spectrometry of Hillenbrand (1997). Stellar masses and ages depend on the model assumptions of the evolutionary tracks adopted in our study (D'Antona \& Mazzitelli 1997). These quantities will systematically change with differing assumptions regarding the equation of state, mixing length theory, accretion, rotation, and the internal magnetic field (D'Antona et al. 2000; Palla 2001). The effects of even modest observational error on parameters derived from evolutionary tracks, especially stellar age, may be significant: an uncertainty $\Delta T_{\text {eff }}= \pm 100 \mathrm{~K}$ and $\Delta L_{\text {bol }}= \pm 0.1$ produces fractional errors around $\Delta \log M= \pm 0.1 \%$ and $\Delta \log t=$ \pm 0.5 (Siess 2001). Rotational periods generally have almost no statistical uncertainty, but sometimes suffer large errors if the wrong peak in a periodogram is chosen. A few stars in our sample with discrepant reported periods of this type are listed in the notes to Table 1 of F02a.

We thus expect scatter in various stellar properties, particularly age, due to observational error, plus possible systematic errors in properties due to model assumptions. In most cases, the latter may produce offsets or stretching of the plotted axes, but will not affect overall strength of a correlation. The greatest danger would arise if both the X-ray

\footnotetext{
${ }^{7}$ We omit JW 991 because of its low probability of cluster membership (Jones \& Walker 1988).
}

luminosity and another property of interest were mutually dependent on magnetic field generation, producing spurious correlations. However, this problem does not appear to be present: PMS model interiors with magnetic fields tends to have cooler surfaces which would yield lower inferred masses (D'Antona et al. 2000). In contrast, we find below $(\S 4.3)$ that Orion stars with stronger magnetic activity have higher rather than lower masses than those with weak activity.

\section{X-RAY DEPENDENCIES ON STELLAR PROPERTIES}

We present here empirical results relating the X-ray emission, viewed as an indicator of magnetic activity, to the bulk properties of the ONC PMS stars: bolometric luminosity, mass, age, presence of disk, and surface rotation. In some cases we elucidate long-standing relationships found from past studies ( $\{2.1 .3)$, while in other cases we reveal new phenomenology. The findings are summarized in $\S 6.1$.

\subsection{X-Ray and Bolometric Luminosities}

With a sample population far larger than previously available, we can now see why a correlation between $L_{s}$ and $L_{\text {bol }}$ has been seen in past studies of PMS stellar populations but with inconsistent quantitative results (e.g., Walter \& Kuhi 1981; Feigelson et al. 1993; Casanova et al. 1995; Gagné et al. 1995; Stelzer \& Neuhäuser 2001; Preibisch \& Zinnecker 2002; Getman et al. 2002). Figure $2 a$ shows a broad correlation over 3 orders of magnitude, roughly consistent with the linear relationship $\log L_{t} \simeq 29.8+\log L_{\mathrm{bol}}$ $\mathrm{ergs} \mathrm{s}^{-1}$ or, as seen in Figure $4 c, \log L_{t} / L_{\mathrm{bol}} \simeq-3$.8. However, because of the selection bias against X-ray-faint lowmass stars (§3.3), it is likely that the median X-ray luminosity at low $L_{\text {bol }}$ values is overestimated here, leading to a steeper true relation. For example, the data could be modeled as $L_{t} \propto L_{\mathrm{bol}}^{2}$ with a saturation limit at high luminosities. Although difficult to quantify because of the scatter and bias, examination of the notches in the box plot (Fig. 2b) shows that the overall correlation has very high statistical significance.

Whatever the underlying relationship between X-ray luminosity and $L_{\mathrm{bol}}$, a great deal of scatter is present. ${ }^{8} \mathrm{At}$ any given $L_{\mathrm{bol}}$ value, the dispersion in $L_{t}$ or $L_{t} / L_{\mathrm{bol}}$ is such that half of the stars lie greater than a factor of 3 away from the predicted value, and some are discrepant by more than an order of magnitude. This dispersion must be astrophysical in origin, as it is too large to arise from observational or absorption effects. It is likely that X-ray flaring is a major contributor to this dispersion, but other dependencies on other variables may also be important.

\subsection{X-Ray Emission and Stellar Size}

As most T Tauri stars have similar surface temperatures $T_{\text {eff }}$, bolometric luminosity is closely related to stellar surface area via $L_{\mathrm{bol}}=4 \pi R^{2} \sigma T_{\mathrm{eff}}^{4}$ where $\sigma$ is the StefanBoltzman constant. X-ray luminosities that scale with $L_{\text {bol }}$ will therefore also scale with stellar surface area, radius, and volume. Figure 3 shows one of the relationships: X-ray

\footnotetext{
${ }^{8}$ The outliers with high $L_{\mathrm{bol}}$ and very low $L_{t}$ are discussed in $\S 4.3$; they are most vividly seen in Figure $4 c$. Similar outliers are found by Preibisch \& Zinnecker (2002) from Chandra observations of the IC 348 young stellar cluster.
} 


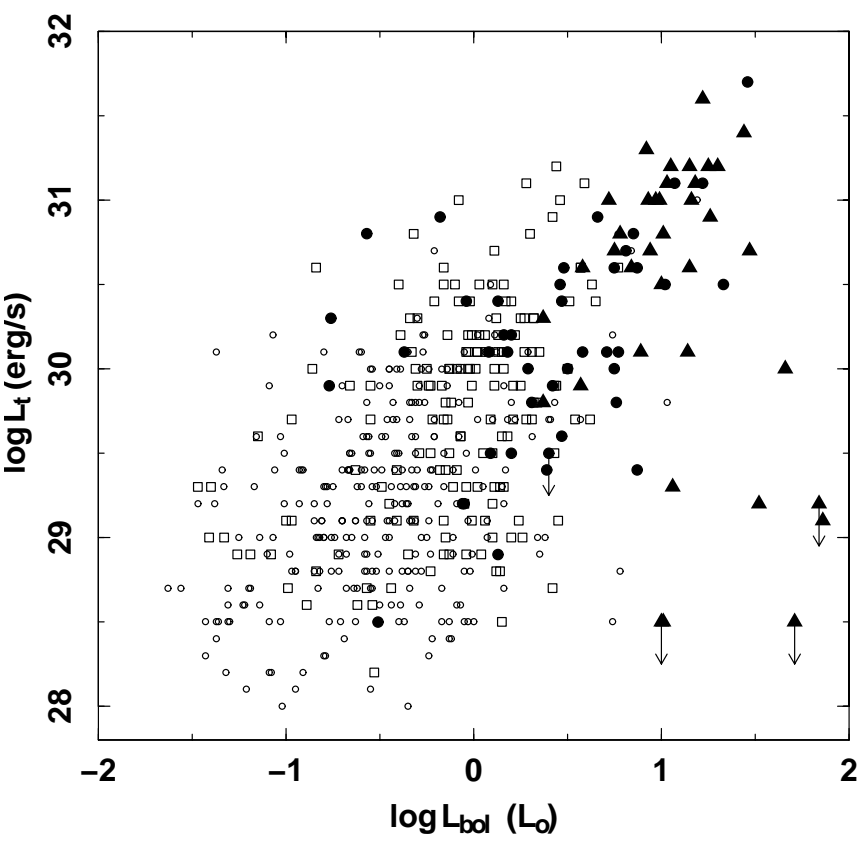

FIG. $2 a$

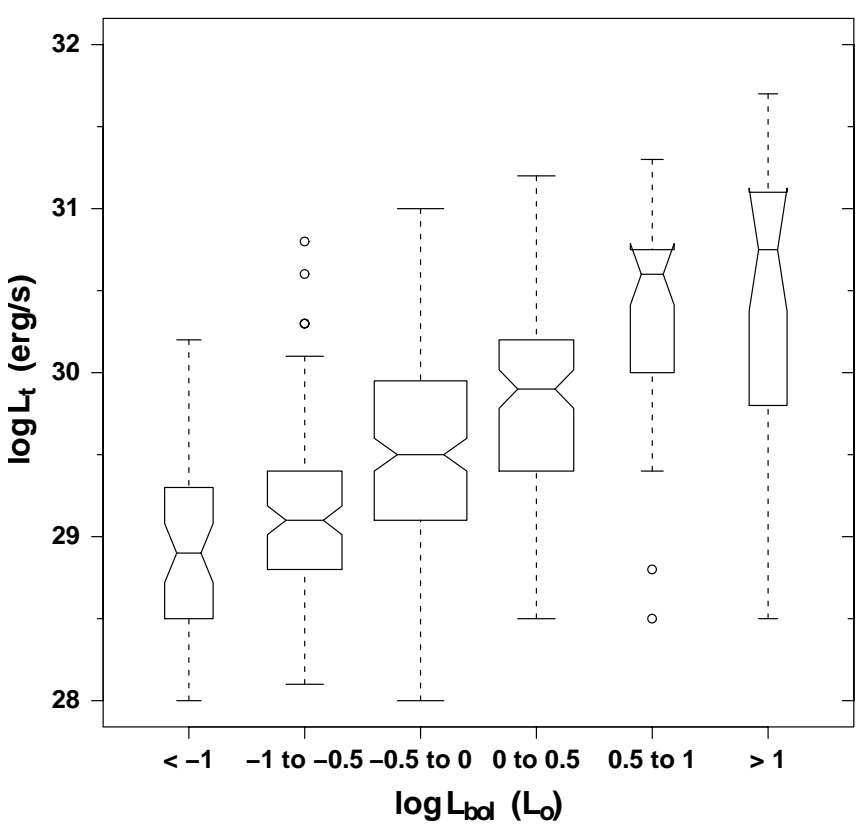

FIG. $2 b$

FIG. 2.-Relationship between PMS X-rays and bolometric luminosities: (a) scatter plot of $\log L_{t}$ and $\log L_{\text {bol }}$, and $(b)$ box plot of $\log L_{t}$ and $\log L_{\text {bol }}$. The scatter plot symbols here and in later figures are coded by mass as follows: $1.4<M<3.0 M_{\odot}$ (large filled triangles); $0.7<M<1.4 M_{\odot}$ (large filled circles); $0.25<M<0.7 M_{\odot}$ (open squares); and $M<0.25 M_{\odot}$ (small open circles). The four X-ray nondetections with $M>0.7 M_{\odot}$ are shown with arrows.

emission compared with stellar volume in units of $V_{\odot}=4 \pi R^{3} / 3$. Recognizing that the median levels for the smallest stars are probably overestimated because of nondetections $(\S 3.3)$, we find that X-ray luminosity scales roughly as $L_{t} \propto V^{2 / 3} \propto R^{2}$.

A similar, but considerably steeper, activity-radius effect has been found in a sample of dM 1V main-sequence stars by Houdebine \& Stempels (1997). They find that $\mathrm{H} \alpha$, Ca II $\mathrm{H}$ and $\mathrm{K}$, and soft $\mathrm{X}$-ray activity indicators scale with absolute magnitude, which, for constant surface temperature, itself scales with radius, surface area, and volume. Stated another way, magnetically active early dMe stars are more luminous, and hence larger, than less active dM stars. The $\mathrm{dM}$ X-ray sample is small and suggested a relationship around $L_{s} \propto R^{7}$.

\subsection{X-Ray Emission and Stellar Mass}

Figure 4 shows scatter diagrams and box plots of X-ray emission as a function of stellar mass. A comparison of Figures $4 a$ and $4 b$ to Figure 2 shows that mass is as strong a predictor for X-ray emission as bolometric luminosity, although the X-ray/mass relation has only occasionally been noticed in past studies, with discrepant quantitative results (e.g., Feigelson et al. 1993; Neuhäuser et al. 1995; Preibisch \& Zinnecker 2002). Given a strong $L_{t}-L_{\text {bol }}$ correlation, a similar $L_{t}-M$ relation is expected from a coeval PMS population given the tilt of the isochrones with respect to the isomass lines in the HR diagram. The relationship appears steeper than linear, roughly consistent with $\log L_{t} \simeq 30.2+1.5 \log M$ ergs s${ }^{-1}$, but again we recall the selection bias $(\S 3.3)$ that should increase the slope of this relation at low masses. This is consistent with the recent Chandra-based result $\log L_{t}=30.10+1.97( \pm 0.24) \log M$ ergs $\mathrm{s}^{-1}$ derived by Preibisch \& Zinnecker (2002) in the IC
348 young stellar cluster over a mass range similar to that considered here. ${ }^{9}$

The $\log L_{t} / L_{\text {bol- }} M$ diagram (Fig. $4 c$ ) dramatically reveals an effect distinct from the general $L_{t}-M$ relationship: X-ray emission from the higher mass stars in the sample with $2.0<M<3.0 M_{\odot}$ has an enormous dispersion. It is possible that, for $M>2 M_{\odot}$, the ONC population can be divided into two classes. The majority of these $2-3 M_{\odot}$ stars have $-5<\log L_{t} / L_{\text {bol }}<-3$, like virtually all lower mass stars, while a minority ${ }^{10}$ show $-7<\log L_{t} / L_{\text {bol }}-5$. The latter low X-ray emissivities are ubiquitous for the intermediatemass $3<M<30 M_{\odot}$ ONC stars (see Fig. $12 a$ in F02a). Two interpretations of this difference in X-ray behavior of intermediate- and low-mass PMS stars are outlined in $\S 6.2$.

\subsection{X-Ray Emission and Circumstellar Disks}

From the very beginning of X-ray studies of PMS populations, most studies found that accretion and outflows associated with "classical" T Tauri star-disk interactions

\footnotetext{
9 The observed $L_{t}$-mass correlation may be affected by unresolved binarity, which is likely to be present in over half of the ONC "stars" under study (Mathieu 1994). However, it seems unlikely that the effect is very significant for most of the T Tauri stars shown here. If fainter secondary components were responsible for the X-ray emission, then the low-mass systems should show as wide a spread in $L_{t}$ as high-mass systems, and the $L_{t}$-mass correlation would be weak. A ROSAT study of nearby T Tauri stars confirms that the X-ray emission of primaries dominates over the secondaries in resolved wide binaries (König, Neuhäuser, \& Stelzer 2001). Note, however, that we do believe binarity may be important for the interpretation of X-ray emission from higher mass $\left(M<2 M_{\odot}\right)$ stars.

10 There is no indication these X-ray-weak stars are foreground interlopers, as their proper motions have $98 \%-99 \%$ probabilities of cluster membership (Hillenbrand 1997). These stars, however, are older than most ONC stars; it possible that both mass and age are involved in their unusually low magnetic activity. Note that weak evidence for a decay in X-ray emission as PMS stars age was reported for $0.7<M<1.4 M_{\odot}$ stars by $\mathrm{F} 02 \mathrm{~b}$, and is discussed again in $\S 4.5$.
} 


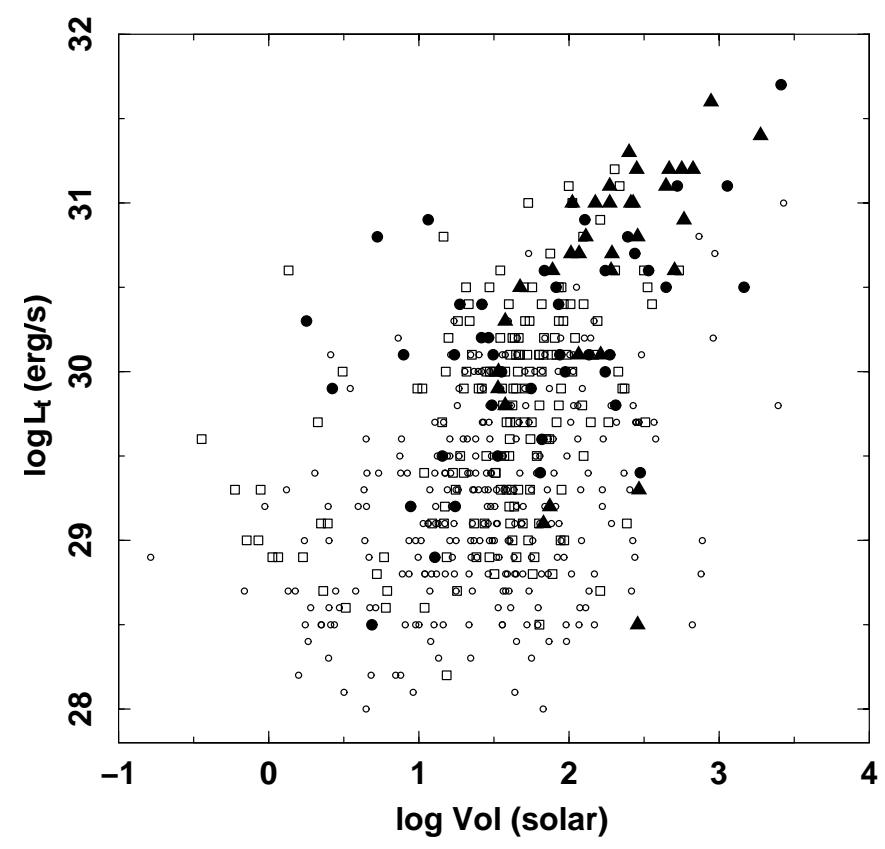

FIG. $3 a$

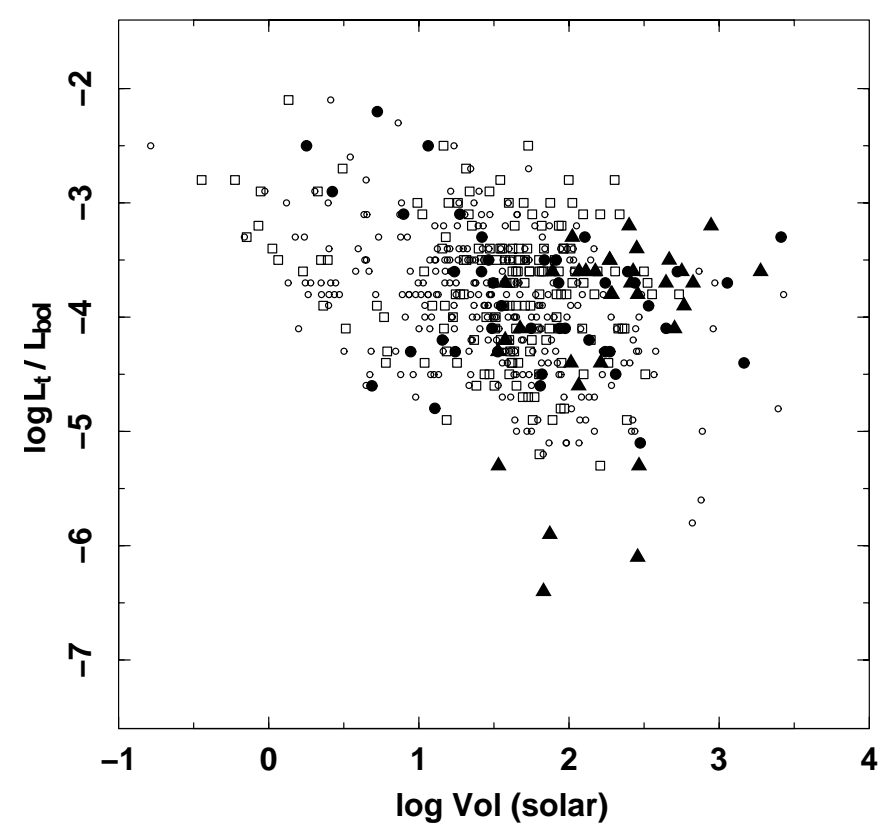

FIG. $3 c$

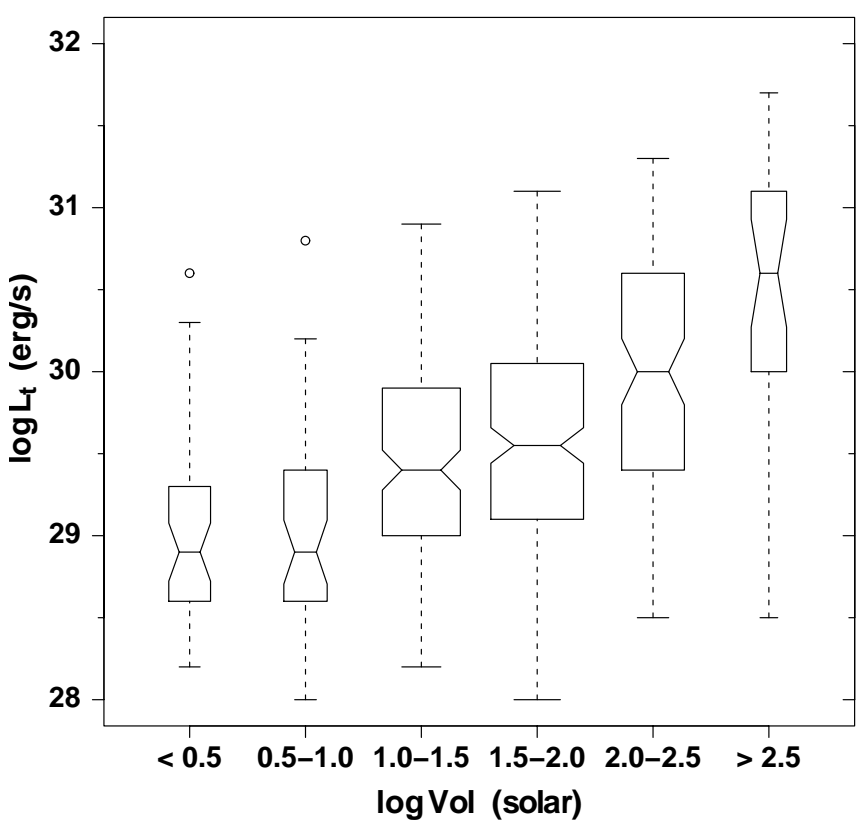

FIG. $3 b$

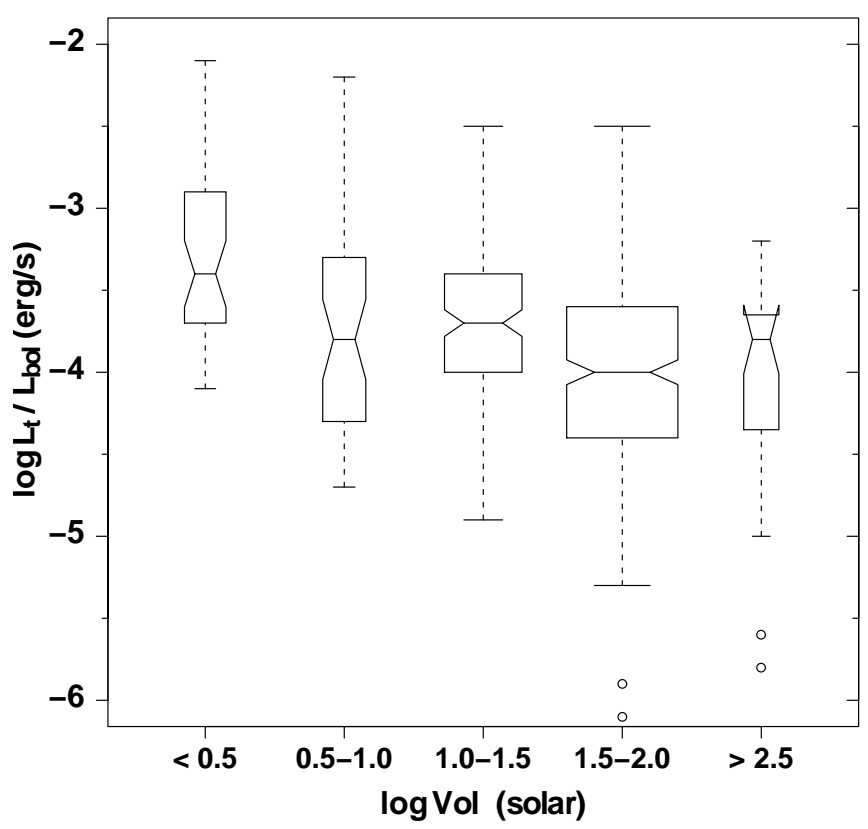

FIG. $3 d$

FIG. 3.-Relationship between PMS X-rays and stellar volumes: (a) scatter plot of $\log L_{t}$ and $\log V$, (b) box plot of $\log L_{t}$ and $\log V$, (c) scatter plot of $\log L_{t} / L_{\mathrm{bol}}$ and $\log V,(d)$ box plot of $\log L_{t} / L_{\mathrm{bol}}$ and $\log V$. See Fig. 2 for symbol definitions.

were not essential ingredients for elevated X-ray levels. This is often shown as an absence of correlation between X-ray and $\mathrm{H} \alpha$ emission when a full PMS population of weak-lined and classical $\mathrm{T}$ Tauri stars is treated, although an X-ray $/ \mathrm{H} \alpha$ correlation may be present within the weak-lined $\mathrm{T}$ Tauri stars alone where both arise from magnetic activity (e.g., Montmerle et al. 1983; Feigelson et al. 1993; Damiani \& Micela 1995; Casanova et al. 1995; Gagné et al. 1995). In contrast, some studies find that weak-lined T Tauri stars (defined by weak $\mathrm{H} \alpha$ emission) are an order of magnitude more X-ray luminous than classical T Tauri stars (Neuhäuser et al. 1995; Stelzer \& Neuhäuser 2001). However, this result may be influenced by misclassifications and incompleteness in the sampling of X-ray-faint weak-lined T Tauri stars, in contrast to the good optical sampling of X-ray-faint classical T Tauri stars (Preibisch \& Zinnecker 2002).

We consider here the photometric near-infrared excess measure $\Delta(I-K)>0.3$ as a discriminant of the presence of a disk, which is not necessarily the same as strong optical emission lines, which indicate the presence of an accreting disk. Figure 5 shows no important relationship between $\mathrm{X}$-ray emission and the presence of a disk. (Another view of this result appears in the middle panel of Fig. 10 in F02a.) Figure $5 c$ shows that mass, which is a strong correlate of $L_{t}$, is not an important confounding variable in this result. 


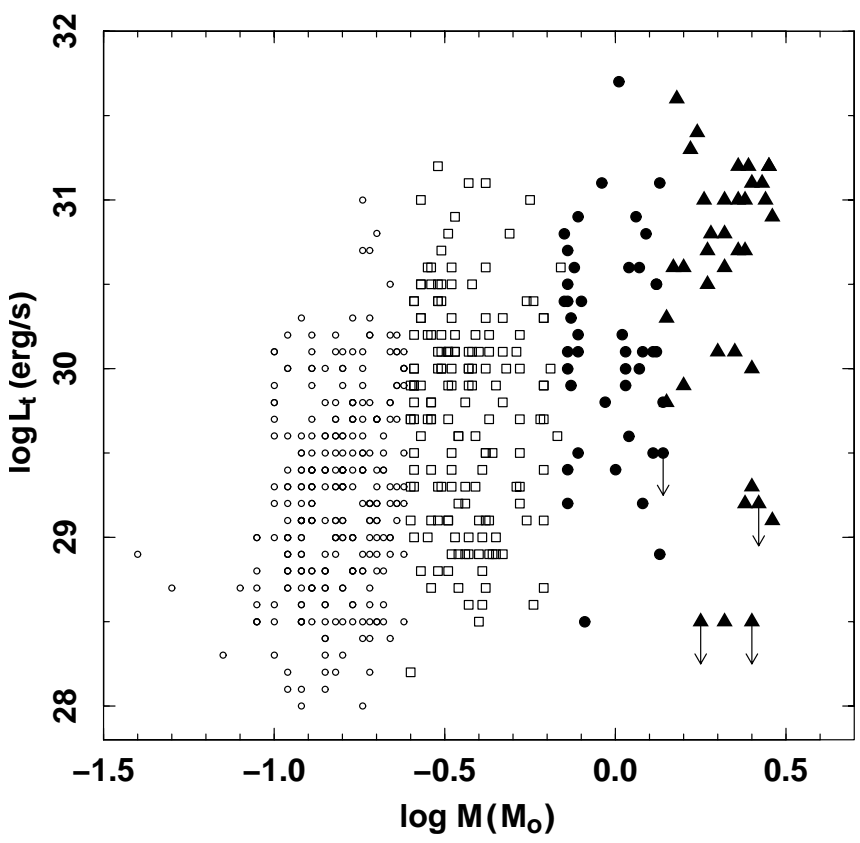

FIG. $4 a$

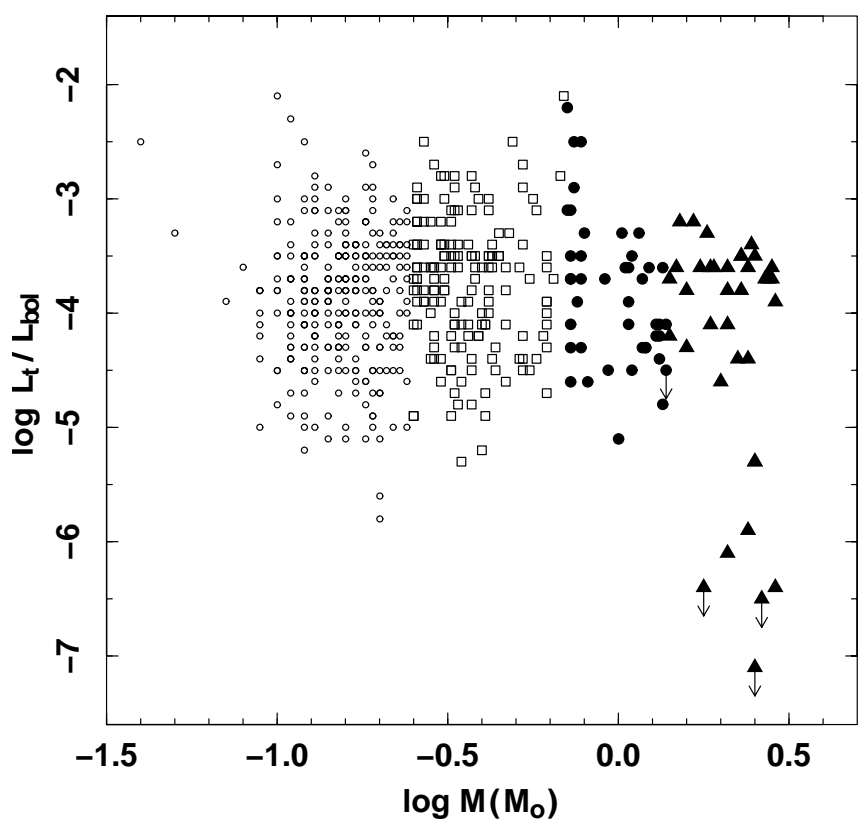

FIG. $4 c$

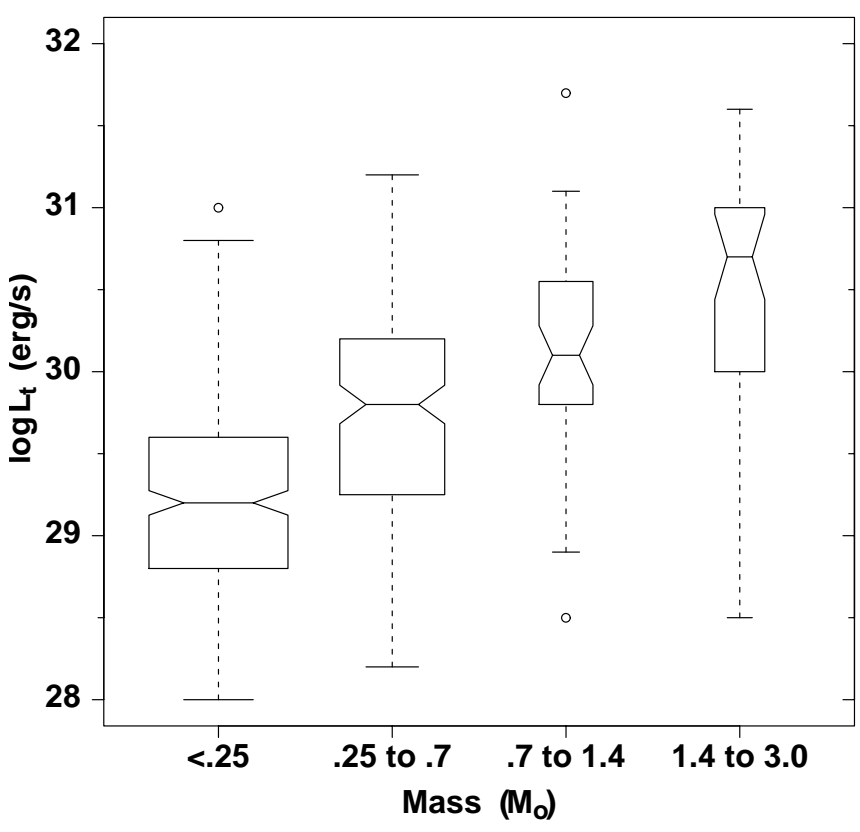

FIG. $4 b$

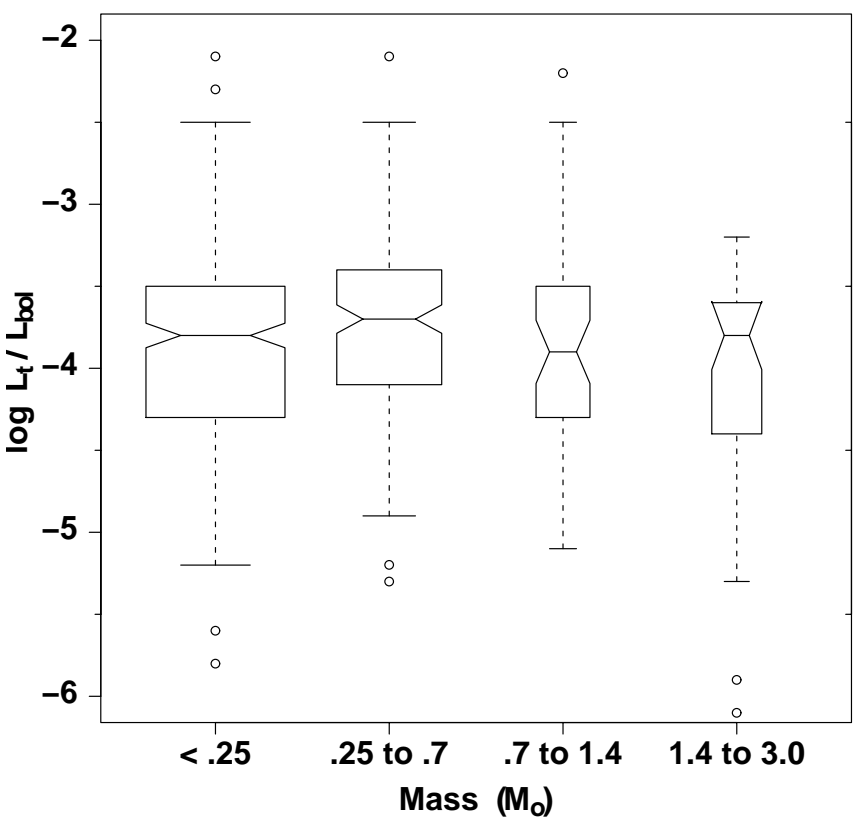

FIG. $4 d$

Fig. 4.-Relationship between PMS X-rays and stellar masses: (a) scatter plot of $\log L_{t}$ and $\log M$, (b) box plot of $\log L_{t}$ and $\log M$, (c) scatter plot of $\log L_{t} / L_{\mathrm{bol}}$ and $\log M,(d)$ box plot of $\log L_{t} / L_{\mathrm{bol}}$ and $\log M$. See Fig. 2 for symbol definitions.

\subsection{X-Ray Emission and Stellar Age}

Low-mass stars evolve in many respects during their descent along the Hayashi tracks: the star contracts; brief periods of deuterium and lithium burning occur; a radiative core forms and grows although most of the star is convective; and star-disk interaction declines or terminates, perhaps releasing the star from rotational coupling with the disk. While most ONC stars appear to have formed within the past $2 \mathrm{Myr}$, a tail of stellar ages appears to extend beyond $10 \mathrm{Myr}$, although it is not clear that these ages are accurate. It is possible that the older Myr stars in the field are interlopers from the older Orion
Ia-c OB associations (see discussion in Hillenbrand 1997; Hartmann 2001).

Past study of the evolution of X-ray emission along the Hayashi tracks has been limited and somewhat confusing. In ROSAT studies of individual PMS clusters, Feigelson et al. (1993) report a tentative drop of soft-band $L_{s}$ from $<1$ to $10 \mathrm{Myr}$, while Neuhäuser et al. (1995) report a rise with age. Kastner et al. (1997) collect average soft X-ray levels for stars from several clusters of different ages and find that $\left\langle\log L_{s} / L_{\mathrm{bol}}\right\rangle$ rises an order of magnitude over tens of Myr. We caution that comparisons of mean X-ray luminosities of different clusters is subject to systematic error due to different X-ray sensitivities and different levels of prior 


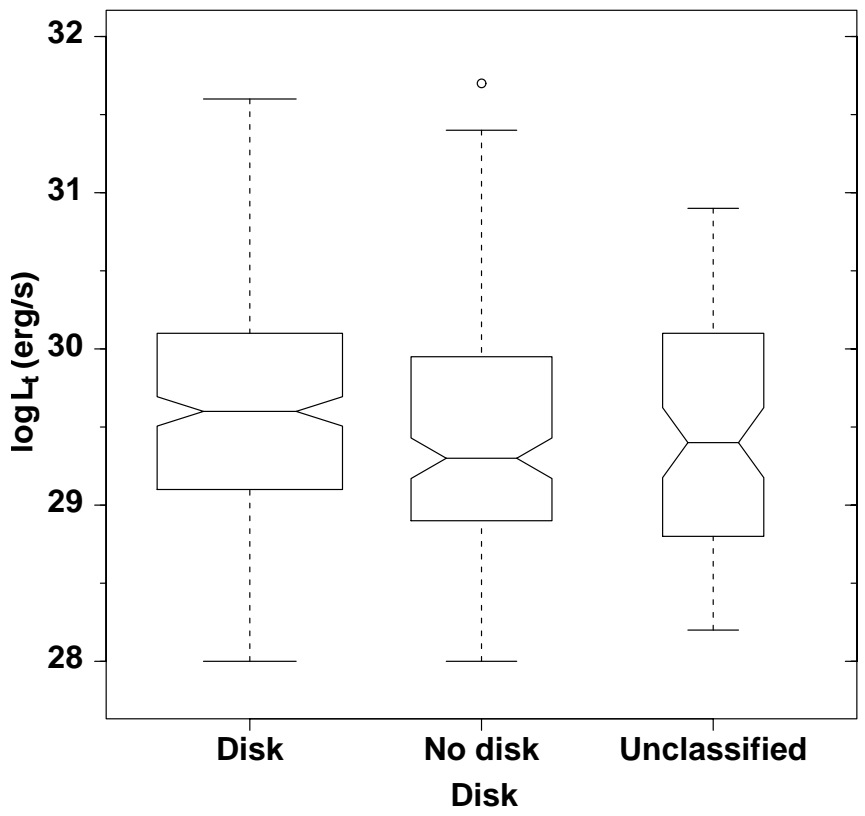

FIG. $5 a$

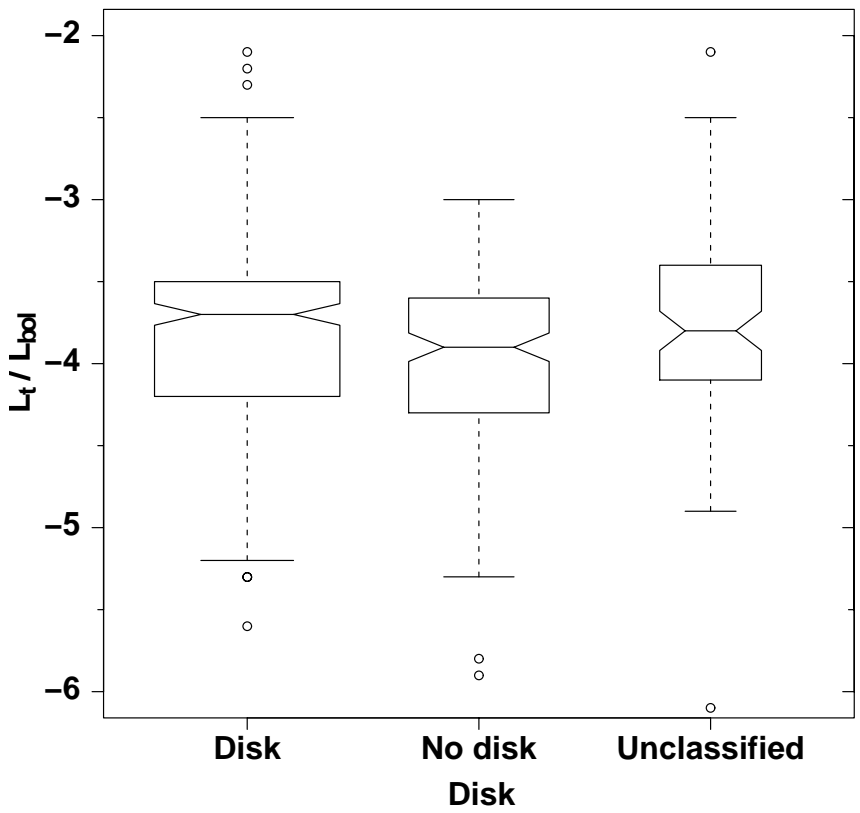

FIG. $5 b$

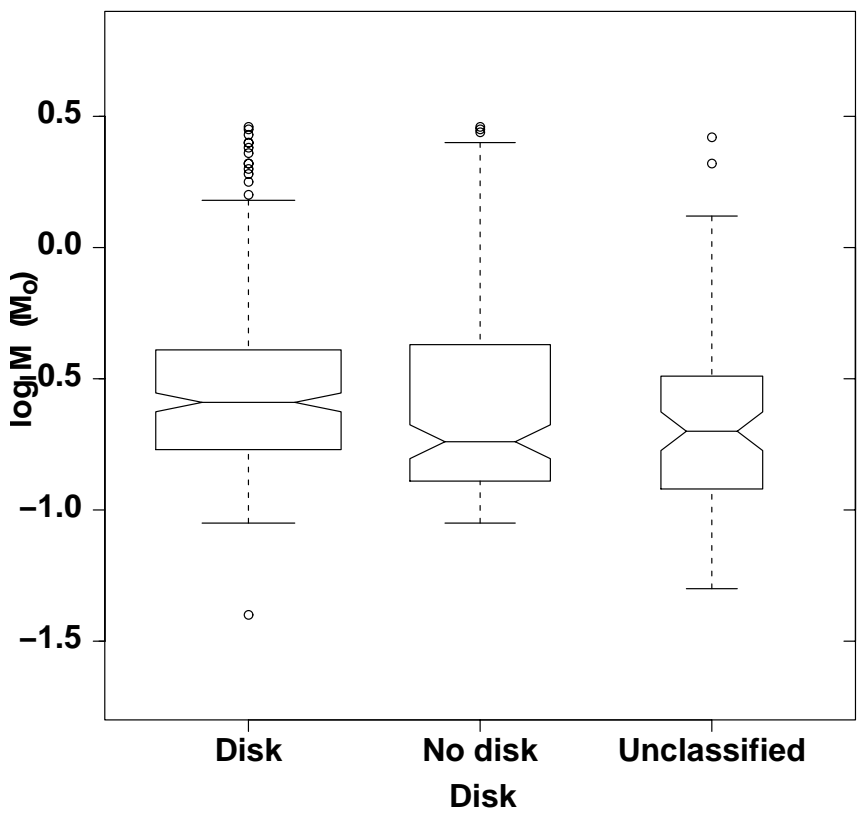

FIG. $5 c$

Fig. 5.-Relationship between PMS X-rays and circumstellar disks: box plots of $(a) \log L_{t},(b) \log L_{t} / L_{\mathrm{bol}}$, and (c) mass vs. disk indicator. See Fig. 2 for symbol definitions.

knowledge of the cluster memberships. A rise in X-ray emissivity with PMS age is consistent with a model of stellar angular momentum evolution in which surface rotation (and presumably the internal magnetic dynamo efficiency) rises as star-disk rotational coupling ends and the star contracts (Bouvier et al. 1997a; Barnes et al. 2001). This model is supported by study of the $\eta$ Cha cluster, a recently identified older PMS cluster with $t=9$ Myr stars, in which nearly all have unusually short rotational periods and high X-ray luminosities around $\log L_{s} / L_{\mathrm{bol}} \simeq-3$ (Mamajek, Lawson, \& Feigelson 2000; Lawson et al. 2001).

Figure 6 shows the X-ray/age relationship found for the ONC sample discussed here. Recall that ages were estimated from the evolutionary tracks of D'Antona \& Mazzitelli
(1997) based on the photometry and spectroscopy of Hillenbrand (1997), and that we truncate all extremely young inferred ages at $0.3 \mathrm{Myr}$. Figures $6 a$ and $6 b$ reveal a small but statistically significant decline in X-ray luminosity from a median level of $\log L_{t} \simeq 29.6 \mathrm{ergs} \mathrm{s}^{-1}$ for ages less than $1 \mathrm{Myr}$ to $\log L_{t} \simeq 29.2 \mathrm{ergs} \mathrm{s}^{-1}$ for ages above $10 \mathrm{Myr}$. A similar but steeper drop in $L_{t}$ is found when the $0.7-1.4 M_{\odot}$ solar analogs are considered alone (F02b). We also note that the dispersion in X-ray luminosities decreases monotonically with age from more than 3 to 2 orders of magnitude.

Figures $6 c$ and $6 d$ show that this fall in X-ray luminosity is roughly equal to the decrease in bolometric luminosity from 0.3 to $10 \mathrm{Myr}$, so that the X-ray emissivity $\log L_{t} / L_{\mathrm{bol}}$ is roughly constant at -3.8 . However, a distinctive change 


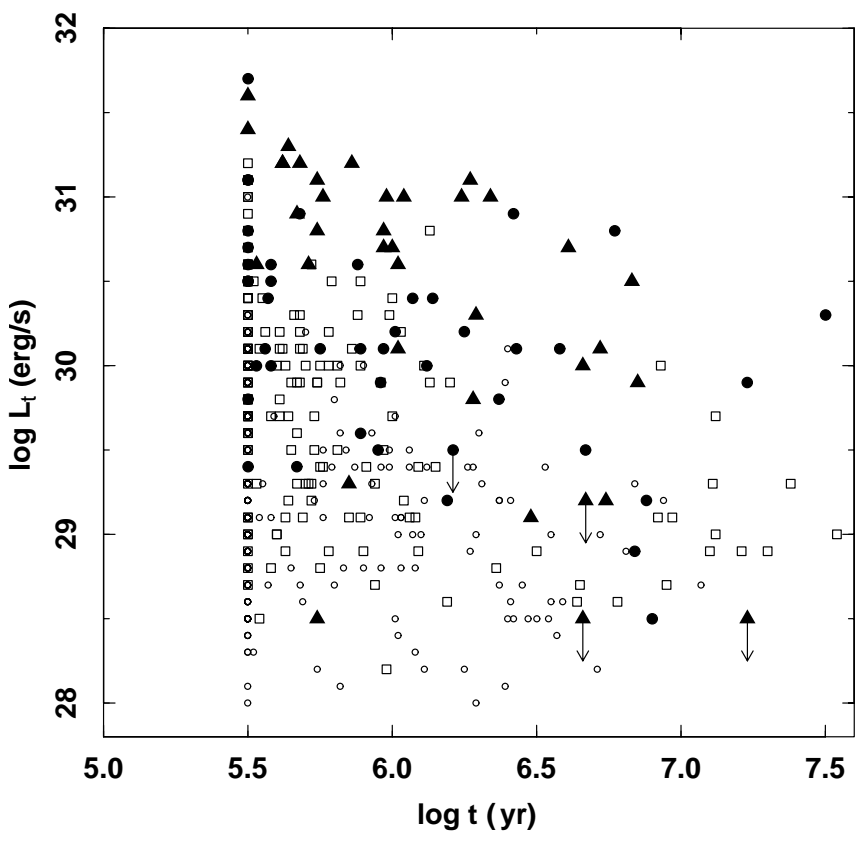

FIG. $6 a$

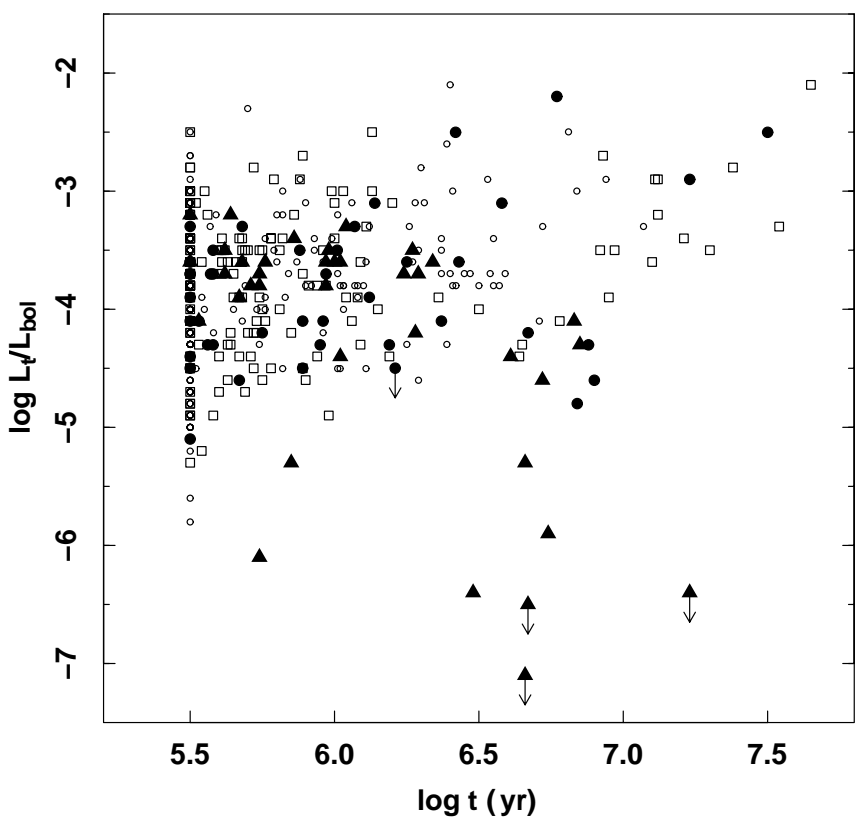

FIG. $6 c$

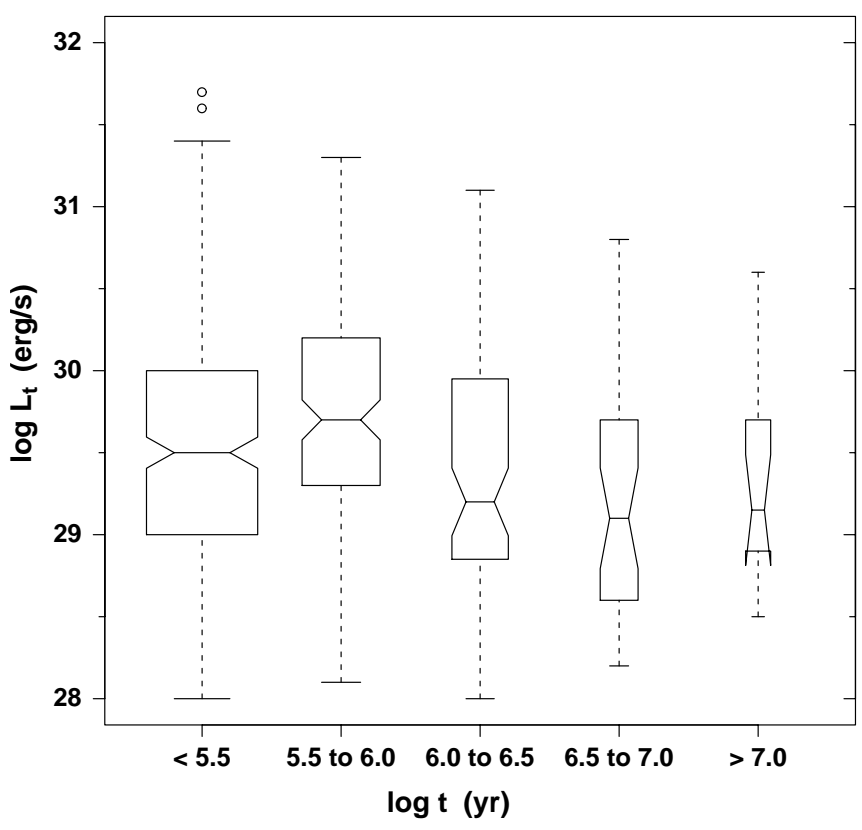

FIG. $6 b$

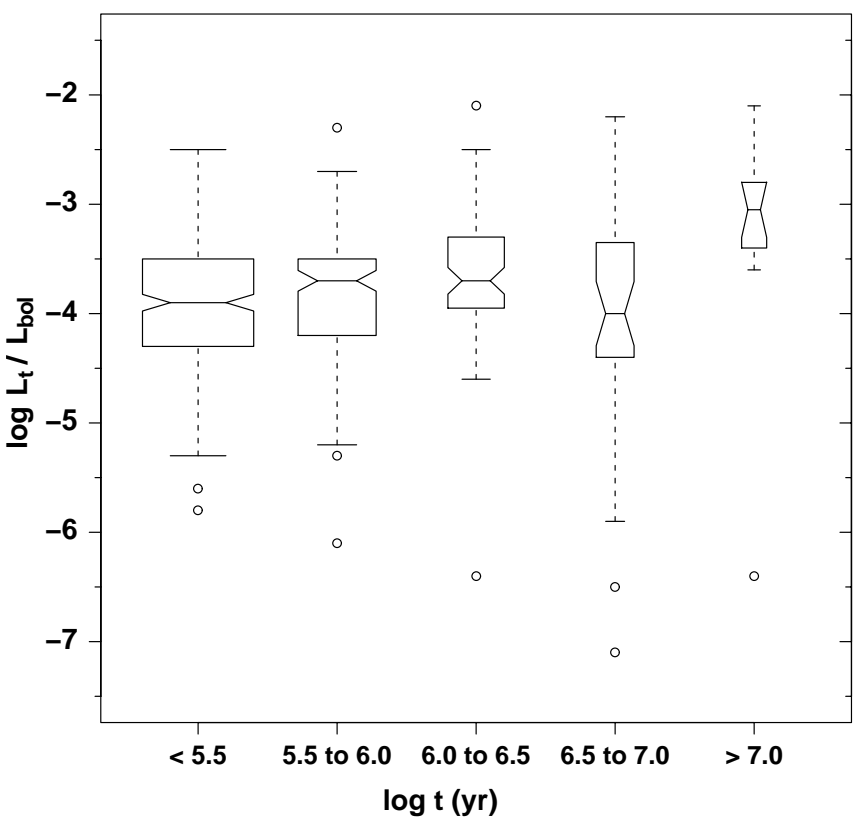

FIG. $6 d$

Fig. 6.- Relationship between PMS X-rays and stellar ages: (a) scatter plot of $\log L_{t}$ and $\log t$, (b) box plot of $\log L_{t}$ and $\log t$, (c) scatter plot of $\log L_{t} / L_{\mathrm{bol}}$ and $\log t,(d)$ box plot of $\log L_{t} / L_{\mathrm{bol}}$ and $\log t$. See Fig. 2 for symbol definitions.

is seen among the oldest ONC stars: with the exception of a single intermediate-mass outlier (see $\S 4.3$ ), all of the 13 ONC stars with apparent ages between 10 and $30 \mathrm{Myr}$ have unusually high $\mathrm{X}$-ray emissivities, with $\log L_{t} / L_{\text {bol }} \simeq-3$ at the main-sequence saturation level, similar to the $\eta$ Cha finding. There are several possible interpretations for these stars. If they are indeed cluster members and are correctly placed in the HR diagram, they suggest an increase of $L_{t} / L_{\text {bol }}$ with age. However, if they have been erroneously placed on the diagram, perhaps because of an underestimation of their extinction, then $L_{\mathrm{bol}}$ would be higher and the $L_{t} / L_{\text {bol }}$ ratio consistent with the bulk of the ONC PMS stars.

\section{X-RAY EMISSION AND SURFACE ROTATION}

The relationships between $\mathrm{X}$-rays and rotation in ONC PMS stars are shown in Figures 7 and 8. They should be compared to analogous graphs of main-sequence stars shown in Figure 1, which are discussed in $\S 2.1 .1$.

\subsection{X-Rays and Rotational Period}

Figures 7 and 1 immediately show two differences between PMS and main-sequence magnetic activity: a large fraction of ONC stars have considerably stronger X-ray emission than main-sequence stars with similar rotation periods, and the strong main-sequence anticorrelation 


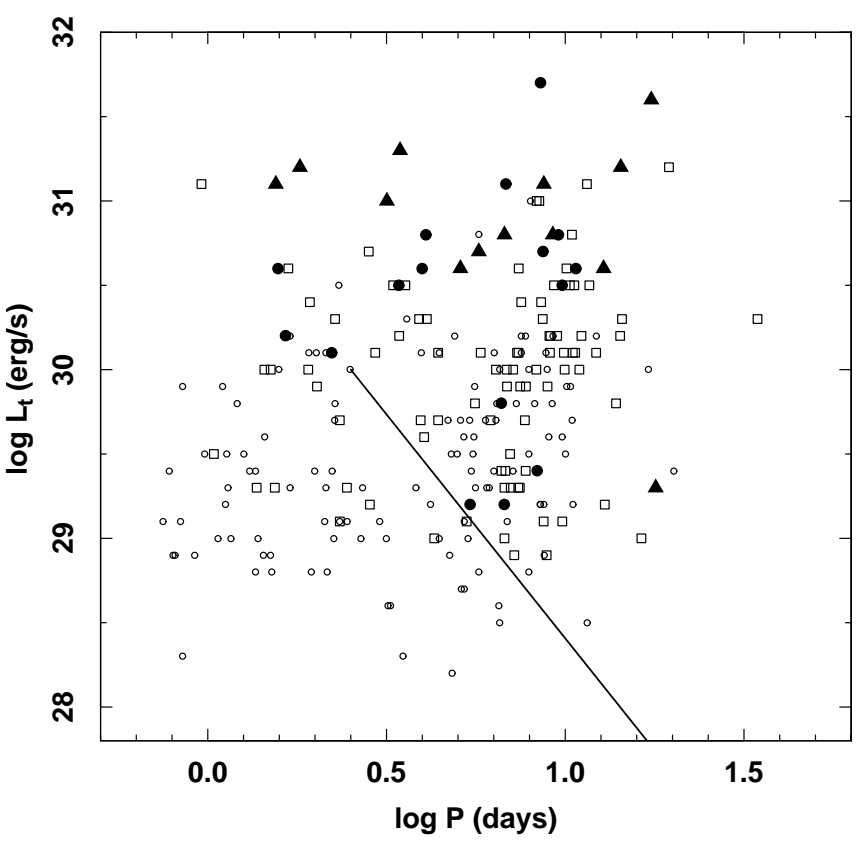

FIG. $7 a$

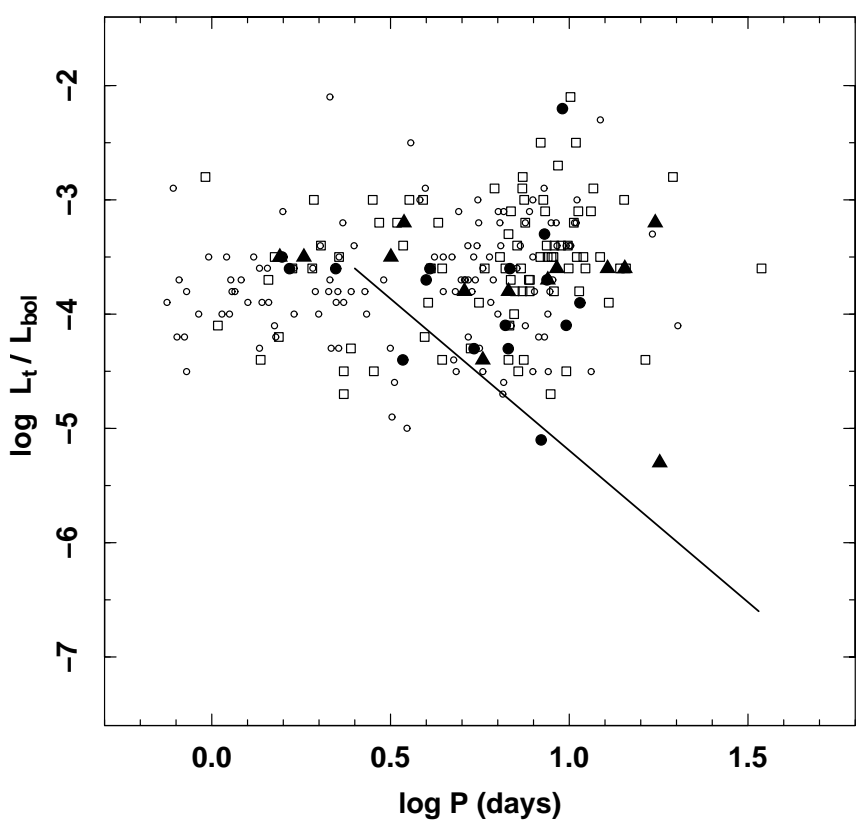

FIG. $7 c$

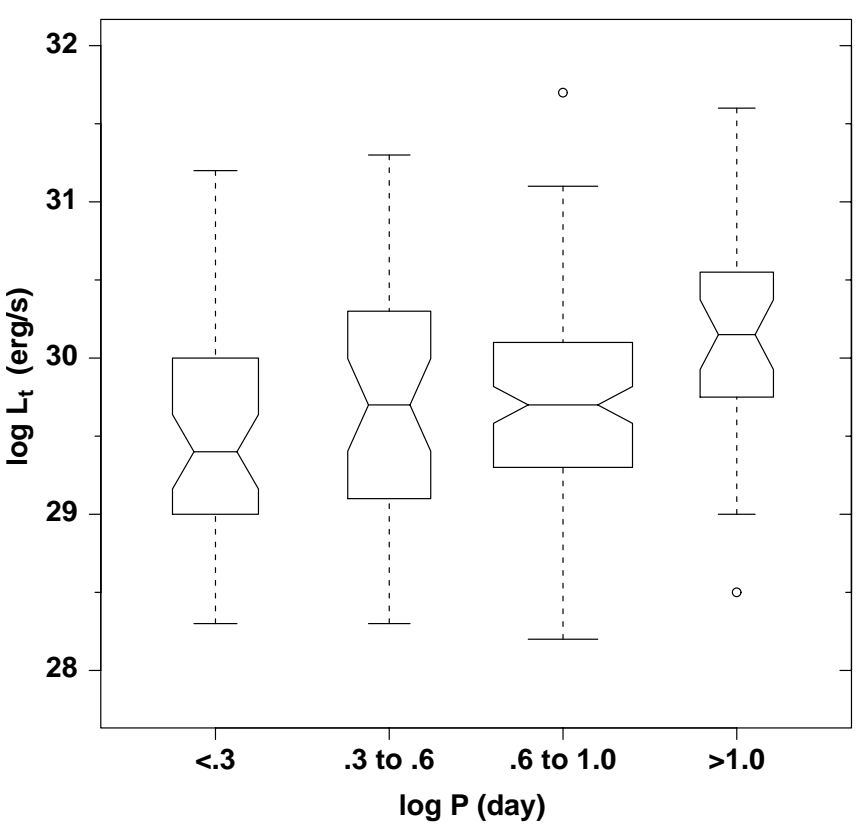

FIG. $7 b$

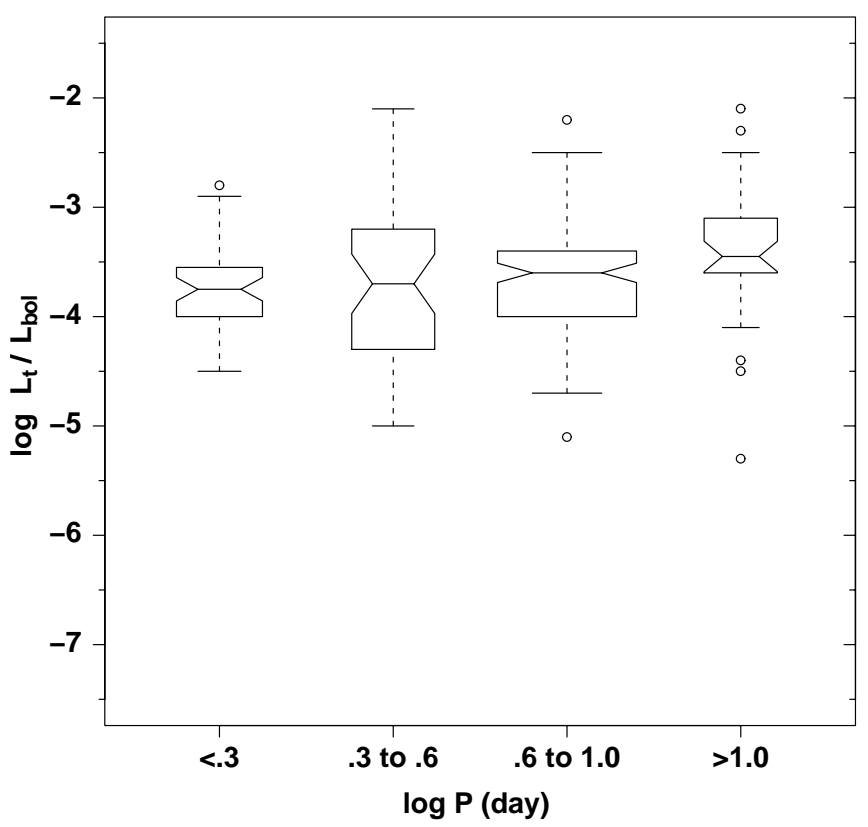

FIG. $7 d$

FIG. 7.- Relationship between PMS X-rays and stellar rotation periods: (a) scatter plot of $\log L_{t}$ and $\log P,(b)$ box plot of $\log L_{t}$ and $\log P,(c)$ scatter plot of $\log L_{t} / L_{\mathrm{bol}}$ and $\log P,(d)$ box plot of $\log L_{t} / L_{\mathrm{bol}}$ and $\log t$. See Fig. 2 for symbol definitions. The lines in panels $a$ and $c$, reproduced from Fig. $1 a$, show the relationships seen in solar-mass main-sequence stars.

between X-rays and period is dramatically absent in the ONC population. ${ }^{11}$ Instead, a correlation in average luminosities with period is marginally present (compare the boxplot notches in Fig. 7b), such that stars with periods $P>10$ days are about 4 times more $\mathrm{X}$-ray luminous on average than stars with $P<2$ days. This trend is in the opposite direction to the strong anticorrelation seen in main-sequence

${ }^{11}$ The locus of ONC stars in Figure $7 c$ also does not follow the roughly parabolic shape, peaking around 1 day, seen in dM stars (James et al. 2000; Mullan \& MacDonald 2001). stars for stars with similar periods; for example, for solarmass stars shown in Figure 1a, the X-ray luminosity of stars with $P>10$ days is $\sim 100$ times smaller than those with $P \simeq 2$ days. The $\log L_{t} / L_{\text {bol }}$ versus $P$ diagram similarly does not show any sign of the steep decline in X-ray luminosity with period seen in main-sequence stars over a similar period range (compare Fig. $7 c$ with Fig. 1a).

Perhaps the most challenging characteristic of this finding to explain is the high X-ray luminosities of very slowly rotating PMS stars. Such stars had been occasionally found in the past; for example, Preibisch (1997) noted that the ONC star JW 157 (= P1659) has a surprisingly high X-ray 


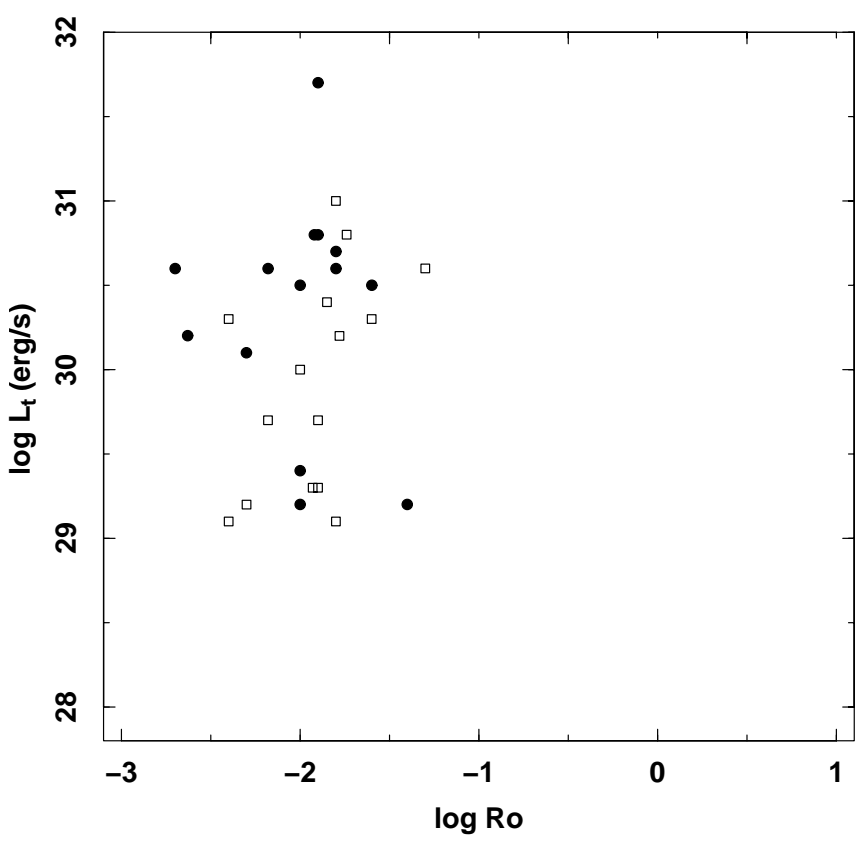

FIG. $8 a$

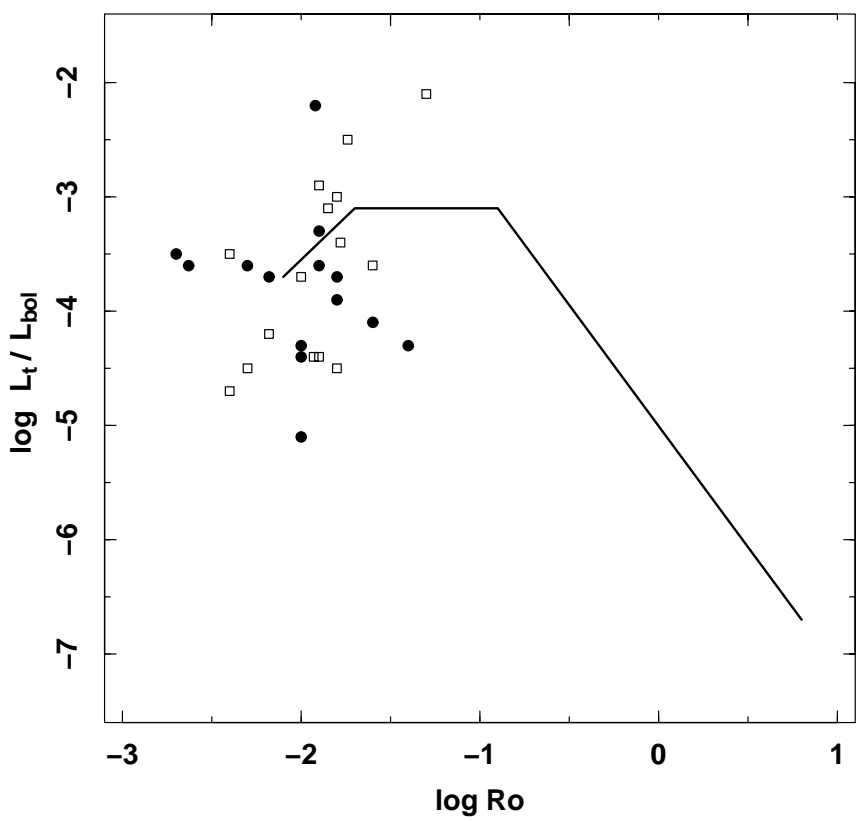

FIG. $8 c$

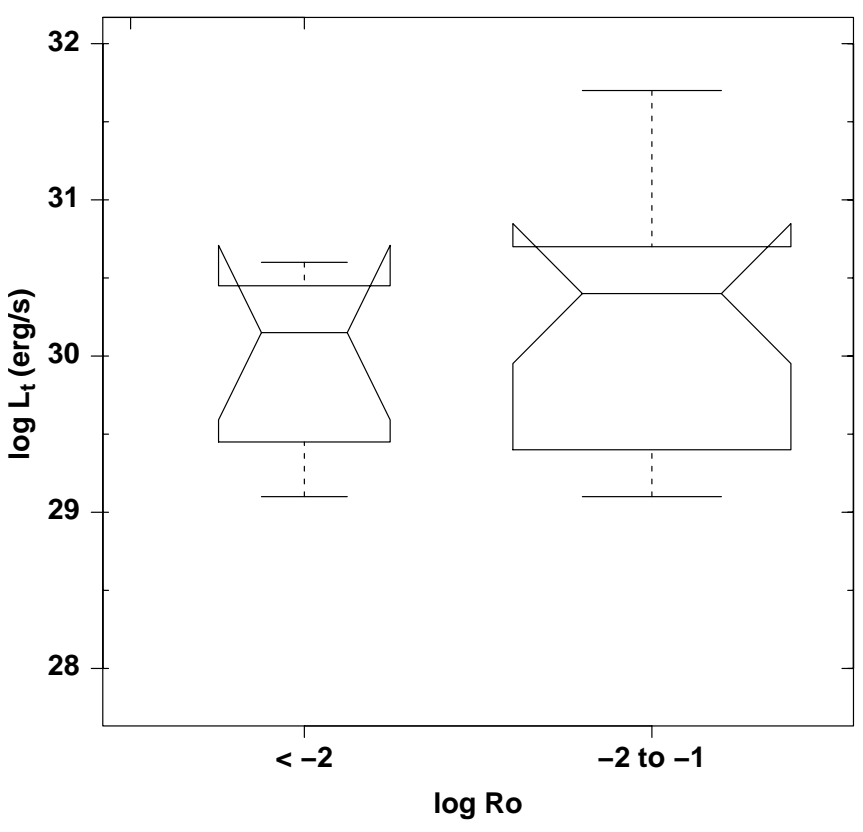

FIG. $8 b$

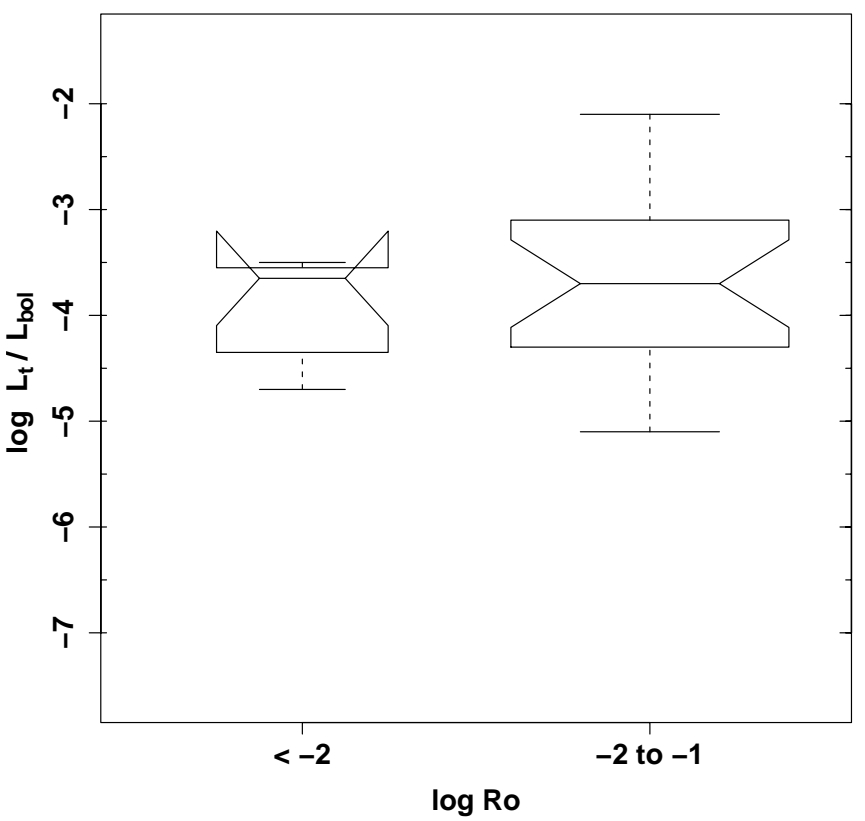

FIG. $8 d$

Fig. 8.-Relationship between PMS X-rays and Rossby number: (a) scatter plot of $\log L_{t}$ and $\log$ Ro, (b) box plot of $\log L_{t}$ and $\log$ Ro, $(c)$ scatter plot of $\log L_{t} / L_{\mathrm{bol}}$ and $\log \mathrm{Ro},(d)$ box plot of $\log L_{t} / L_{\mathrm{bol}}$ and $\log$ Ro. This plot is restricted to stellar masses $0.5-1.2 M_{\odot}$ for which Rossby numbers have been calculated (Kim \& Demarque 1996). See Fig. 2 for symbol definitions. The lines, reproduced from Fig. $1 b$, show the relationship seen in main-sequence stars.

emissivity, $\log L_{s} \simeq 31.5 \mathrm{ergs} \mathrm{s}^{-1}$, for its 17.4 day period, and Lawson et al. (2001) find RECX 10 in $\eta$ Cha has $\log L_{s} / L_{\mathrm{bol}}=-2.9 \operatorname{ergs~s}^{-1}$ with $P=20.0$ days. Both of these are slowly rotating weak-lined $\mathrm{T}$ Tauri stars, although JW 157 appears to be very young $(\log t<5.5 \mathrm{yr})$, while RECX 10 is old $(\log t=7.0 \mathrm{yr})$. The ONC provides a sample of $\simeq 30$ such stars with $P>10$ days and $\log L_{t} / L_{\mathrm{bol}}=-4 \pm 1$ with a wide range of masses.

We recall that some Einstein and ROSAT studies report X-ray/rotation correlations, while others do not $(\S 2.1 .3)$. Perhaps the clearest case that is discrepant from our result is the ROSAT study of Taurus-Auriga PMS stars by Stelzer \& Neuhäuser (2001). They find that, for 39 stars in the soft X-ray band, X-ray emission systematically decreases from $\log L_{s} \simeq 30.6$ to $29.1 \mathrm{ergs} \mathrm{s}^{-1}$ and $\log L_{s} / L_{\text {bol }} \simeq-3.0$ to -4.5 as rotational period increases from $\simeq 1$ to 10 days. We suspect that this discrepancy arises from incompleteness in the Taurus-Auriga sample; it is difficult to define and study the population of this large cloud complex where star formation has occurred in cores dispersed over $500 \mathrm{deg}^{-2}$. First, arguments have been put forward that Taurus-Auriga PMS stellar samples are deficient in both high-mass stars (Walter \& Boyd 1991) and faint low-mass weak-lined T Tauri stars 
(Luhman 2000; Preibisch \& Zinnecker 2002). The effects of such missing stars on an X-ray/rotation diagram is unknown. Second, rotational periods of Taurus-Auriga stars were typically obtained from photometric observations of specific PMS stars with observing sessions spanning $\simeq 10-40$ days (e.g., Bouvier et al. 1986, 1997b) and result in periods for only 39 of 168 stars detected in the study of Stelzer \& Neuhäuser (2001). In contrast, most ONC periods were obtained from observing runs spanning several months or years (Herbst et al. 2000, 2002), and result in periods for 232 of 525 stars in the present ONC study. It is thus possible that an improved study of Taurus-Auriga rotations would show a subpopulation of slow rotators with strong X-ray emission that would remove the X-ray/rotation correlation found by Stelzer \& Neuhäuser (2001).

\subsection{X-Rays and Rossby Number}

It is well known that combining stars of different masses can blur relations between magnetic activity indicators and rotational periods. We address this in two ways. First, examination of individual symbols in the scatter plots in Figure 7, which represent different mass ranges, shows no evidence of the expected decrease in $\mathrm{X}$-ray emission with increasing period within individual mass strata. Second, we consider the X-ray relation to Rossby number, which is very effective in removing massdependent effects in the context of $\alpha-\Omega$ dynamo models (Noyes et al. 1984; Montesinos et al. 2001). As described in $\S 3.2$, we obtain Rossby numbers from the convective turnover times for PMS stars calculated by Kim \& Demarque (1996), recognizing that they assume a single rotation rate and are available only for 0.5-1.2 $M_{\odot}$ stars. The results are shown in Figure 8; Figure $8 c$ is most valuable for its comparison with the main-sequence X-ray/ Rossby number relation (Fig. 1c).

The X-ray/Rossby number plot (Fig. 8b) gives a possible explanation for the absence of the expected X-ray/rotation relation. Because of the very short calculated convective turnover times at the base of the deep convection zones of PMS stars, most ONC PMS stars around $M \sim 1 M_{\odot}$ lie in the supersaturated regime rather than the linear regime where X-ray emission inversely correlates with Rossby number. Extremely long rotation periods around 100 days would be needed to move the ONC stars into the linear regime.

\section{DISCUSSION}

It is valuable to first recognize why this study may achieve results not available to previous observations. For PMS stars, X-rays from reconnection flares are the most easily observed indicator of surface magnetic activity. Optical emission line indicators useful in other types of stars are often confused by lines due to accreted or ejected matter, and the ultraviolet is often obscured by interstellar matter. Doppler imaging and Zeeman effect studies are very valuable for mapping surface fields, but have to date been obtained for only a handful of the brightest $\mathrm{T}$ Tauri stars. X-ray emission, on the other hand, is typically elevated $10^{2} \pm 10^{1}$ times above solar levels during all phases of PMS evolution (Feigelson \& Montmerle 1999). PMS spectra show typical plasma ener- gies around 1-3 keV and are sometimes dominated by plasmas as hot as $\sim 10 \mathrm{keV}$ (F02a), and can therefore been studied even in the presence of considerable interstellar absorption. A $2 \mathrm{keV}$ photon has the same penetrability as a $2 \mu \mathrm{m}$ near-infrared photon, and is comparable to mid-infrared emission above $5 \mathrm{keV}$ (Montmerle \& Grosso 2002). Finally, the ONC provides the largest and best defined PMS sample in the nearby Galaxy in the sense that virtually all members of the cluster appear in the optical/infrared sample with very few contaminants from unrelated objects. The ONC has the largest sample of PMS stars with detailed optical photometric, spectroscopic and rotation measurements. While nearly all earlier X-ray telescopes studied the ONC, only Chandra has the sensitivity and resolution to resolve the crowded cluster core (except for multiple systems). Our observations, for example, achieve more than an order of magnitude greater sensitivity than ROSAT observations of the ONC.

\subsection{Summary of Findings}

In this light, the principal results from examination of bivariate relations between X-ray emission and stellar properties for well-characterized ONC stars are:

1. X-ray luminosities are strongly correlated with several closely coupled stellar properties: bolometric luminosities, stellar size (radius, surface area, and volume), and mass (§ 4.1-4.3). The $\log L_{t}-\log L_{\text {bol }}$ relation, for example, is roughly linear and consistent with an average $\log L_{t} / L_{\mathrm{bol}} \simeq$ -3.8 . This is an order of magnitude below the mainsequence saturation level. The $\log L_{t}$-size relations are consistent with X-ray luminosities scaling linearly with stellar surface area. The dispersion about these relations is high and can be attributed in part to X-ray variability and flaring. The relationship between X-ray luminosities and mass is steeper than linear, and a sharp decrease by more than a factor of 10 in X-ray emissivity $\log L_{t} / L_{\text {bol }}$ is seen in some 2-3 $M_{\odot}$ stars. This drop becomes ubiquitous for ONC stars with $M>3 M_{\odot}$.

2. The presence or absence of a circumstellar disk, as measured by near-infrared photometric excess, appears to have no influence on X-ray luminosities or emissivities $(\S 4.4)$.

3. X-ray luminosities shows a mild decline as stars age and descend the Hayashi track (§ 4.5). Because $L_{\text {bol }}$ also falls, the ratio $\log L_{t} / L_{\mathrm{bol}}$ is constant for $t<10 \mathrm{Myr}$ and may rise to the main-sequence saturation level during $10<t<30 \mathrm{Myr}$.

4. Most importantly for our purposes, X-ray luminosities and emissivities are higher than seen in main-sequence stars for any given rotational period, and show a slight rise with rotational period over the range $0.4 \leq P \leq 20$ days, in contrast to the strong decline seen over the same range in main-sequence stars $(\S 5.1)$. However, the result may be consistent with the main-sequence X-ray/Rossby number diagram, as ONC stars appear to lie in the "supersaturated" regime at low Rossby numbers ( $§ 5.2)$.

\subsection{Implications for Dynamo Models}

Clearly, PMS stars do not exhibit the standard empirical activity-rotation relationships seen in main-sequence stars attributed to an $\alpha$ - $\Omega$ dynamo ( $\S 2.1 .1)$. The X-ray emission 
of an ensemble of mass-stratified PMS stars is unaffected by differences in rotation periods from 0.4 to 20 days, whereas the X-ray emission of main-sequence stars declines by a factor of $10^{3}$ over this same period range. ${ }^{12}$

However, these dramatic differences do not necessarily exclude the application of a standard dynamo (§ 2.2.1) because, based on the limited availability of Rossby numbers for ONC stars, it appears that ONC stars lie in the "supersaturated" regime around $\log \mathrm{Ro} \simeq-2$ (§ 2.1.1). The slight increase in $\log L_{t}$ with $\log P$ seen in the full sample (Fig. $7 b$ ) might represent the rise in X-ray emissivity from the supersaturated to the saturated regime seen in the mainsequence populations.

One argument against an $\alpha-\Omega$ dynamo is the level of saturation: PMS activity shows a log-mean level of $\left\langle\log L_{t} / L_{\text {bol }}\right\rangle=-3.8$, which is $\sim 10$ times below the saturation level seen in main-sequence stars in the $0.5-8 \mathrm{keV}$ band. If the same process of magnetic field generation and eruption is involved in both classes of stars, why should the surface activity differ by so much in a systematic fashion? The finding that X-ray luminosities scale approximately with stellar area (§ 4.2) suggests saturation at the surface, but we cannot eliminate the possibility that X-ray luminosity instead scales with stellar volume, representing a saturation of the internal dynamo.

We are thus led to consider dynamos in which the fields are entirely generated and amplified in the turbulent convection zone that fills all or most of the stellar interior (§ 2.2.2). Such fields may be generated both on small scales, due to turbulence in the convection zone (Durney et al. 1993), and on large scales, driven by a small differential rotation within the interior ( Küker \& Stix 2001; Kitchatinov 2001 and references therein). While a full suite of calculations is not yet available, the solutions appear to be largely independent of the global rotation rate, consistent with the absence of a $\log L_{t}-P$ relation in our findings. These analytical treatments are supported by recent three-dimensional magnetohydrodynamical calculations: fields quickly form and amplify to energy densities above $10 \%$ of the turbulent kinetic energy density in both slab geometries (Thelen \& Cattaneo 2000) and large-scale differentially rotating spherical geometries (Brun 2002). The cause and level of saturation of these distributed dynamos are perhaps not yet clear. ${ }^{13}$

An important constraint on any explanation for PMS $\mathrm{X}$-rays is the change in behavior seen among the more massive 2-3 $M_{\odot}$ stars considered here (§ 4.3). They exhibit an enormous dispersion in X-ray emissivity with some in the

\footnotetext{
${ }^{12}$ The comparison between main-sequence and PMS activity may appear somewhat paradoxical at first glance: PMS X-ray luminosities $\left(\log L_{t}\right)$ are considerably elevated above main-sequence levels, particularly for slow rotators, but PMS X-ray emissivities $\left(\log L_{t} / \log L_{\text {bol }}\right)$ are below the main-sequence saturation level. This discrepancy is easily understood by recalling that PMS stars around $1 \mathrm{Myr}$, as in the ONC, typically have an order of magnitude greater surface area and hence bolometric luminosity than main-sequence stars of the same mass.

${ }^{13}$ One definite prediction of distributed turbulent dynamo models is that magnetic cycles, such as the $22 \mathrm{yr}$ solar oscillation, should be absent. Unfortunately, it will be difficult or impossible to test this in PMS stars using X-rays as the magnetic indicator. First, the flare-dominated X-rays suffer much larger stochastic variability than activity indicators arising from quiescent starspots. Second, stable X-ray instrumentation is rarely available for most than a decade, and X-ray telescope allocations are usually too erratic to give densely sampled time series over many years.
}

$\log L_{t} / L_{\mathrm{bol}}=-4 \pm 1$ range similar to lower mass stars, but others show $\log L_{t} / L_{\mathrm{bol}}=-5 \pm 1$. The emissivity drops further to $\log L_{t} / L_{\mathrm{bol}} \sim-8$ for B stars (F02a). We consider two explanations for this effect, both of which may be operative:

1. Following F02a (their $\S 5.2$ ), these very low X-ray emissivities in intermediate-mass PMS stars may be misleading because of binarity, where a lower mass secondary produces the observed X-rays and the higher mass primary (which dominates $L_{\mathrm{bol}}$ ) is magnetically inactive. The X-ray luminosities of these systems is somewhat higher than the average low-mass PMS ONC stars, implying that the companions have higher than average mass (e.g., $1 M_{\odot}$ rather than $0.3 M_{\odot}$ ). Detailed optical study of the $2-3 M_{\odot}$ population could test the binarity hypothesis.

2. The drop in X-ray emissivity among intermediatemass PMS stars by an order of magnitude (or more if the binary hypothesis is correct) may be linked to structural changes in the stellar interior and consequent changes in dynamo activity. Palla \& Stahler (1993) show that PMS stars with masses above $\simeq 4 M_{\odot}$ arrive at the stellar birthline with radiative interiors undergoing nonhomologous contraction, in contrast to PMS stars below $M \simeq 2 M_{\odot}$ with fully convective interiors undergoing homologous contraction. They predict a narrow range of PMS masses, $2.4<M<3.9 M_{\odot}$ in their canonical model, in which a composite structure of radiative core and convective mantle heated by deuterium burning occurs. The precise boundaries of these structural changes are very sensitive to the initial conditions, so that intermediate-mass $\mathrm{ONC}$ stars with somewhat different ages and accretion histories can have very different structures. These internal structure differences may be reflected in the efficiency of the magnetic dynamo, leading to the wide dispersion of $\log L_{t} / L_{\text {bol }}$ ratios we see in the $2<M<3 M_{\odot}$ mass range (Fig. $4 c$ ). The exact nature of the magnetic fields in these stars is not clear: conceivably, different combinations of a distributed dynamo, tachocline dynamo, or fossil field could be present in different stars with similar masses.

\subsection{Implications for Other Models}

While models of relic and core magnetic fields in PMS stars are not fully developed ( $\S 2.2 .3$ ), our findings do not support these as the source of fields responsible for the observed X-ray emission. We find only a mild temporal dependence of X-ray luminosity on stellar age ranging from $10^{5}$ to $10^{7} \mathrm{yr}(\S 4.5)$, during which time the stellar interior undergoes the important transition to a radiative core. The only hint of a dependence on internal structure is the clear dependence of X-rays on stellar mass. However, we cannot determine whether the $L_{t}-M$ relationship arises from an astrophysical mechanism or as a byproduct of more fundamental relationships like $L_{t} / L_{\mathrm{bol}} \propto$ const, $L_{t} \propto R^{2}$, or $L_{\text {bol }} \propto M$. But if a causal link between magnetic activity and mass is present, it conceivably could arise from the increased trapping of relic fields during the gravitational collapse of more massive stars, or from the increased capture of flux in the radiative core of more massive stars.

Our results also lend little support for models where $\mathrm{X}$-ray emission is associated with a circumstellar disk 
(§ 2.2.4). ${ }^{14}$ This result may have important implications for the physics of the circumstellar disk; in particular, X-ray ionization of disk gas and energetic particle bombardment of disk solids should be lower if the X-rays arise from fields close to the stellar surface than if they arise from the immediate vicinity of the disk (Glassgold et al. 2000; F02b).

Finally, we note that the activity-rotation diagram for PMS stars bears some phenomenological similarity to that obtained for post-main-sequence giants and main-sequence $\mathrm{dM}$ stars (§ 2.1.2). For example, intermediate-mass 2-3 $M_{\odot}$ giants and PMS stars show the same wide range of X-ray luminosities, from $\log L_{s}<28 \mathrm{ergs} \mathrm{s}^{-1}$ to $31 \mathrm{ergs} \mathrm{s}^{-1}$, unaffected by a wide range of rotational velocities (Pizzolato et al. 2000). dM stars show a strong link between $\log L_{s}$ and stellar size (Houdebine \& Stempels 1997). However, as several different models still compete to explain activity in these stars, it is unclear whether phenomenological similarities between the magnetic activity of PMS, $\mathrm{dM}$, and giant stars are astrophysically meaningful.

\section{CONCLUDING COMMENTS}

With the greatly enlarged sample provided by the ONC, observational constraints on the origins of magnetic activity in low-mass PMS stars are more quantitatively and securely established than previous results. However, at present we cannot establish a definitive link between our findings and a unique theory of magnetic field generation in PMS stars.

There are two sources of uncertainty. First, we encounter a degeneracy between the physical properties correlated with X-ray emission. Examination of the evolutionary tracks in the HR diagram readily shows that bolometric luminosity, radius, mass, and age are mutually dependent in a systematic fashion. We thus cannot confidently extract from statistical studies alone which property is astrophysically responsible for the magnetic activity we detect.

Second, theoretical models have often not been sufficiently developed to compare with our empirical findings; additional theoretical calculations are clearly necessary. For example, calculation of Rossby numbers (as in Kim \& Demarque 1996) for each star in our sample using its specific mass, age and rotation, would populate the X-ray/Rossby number diagram (Fig. 8) and possibly reveal new constraints and trends. It would also be very useful if PMS dynamo models involving $\alpha-\Omega, \alpha-\alpha$, and other distributed field generation processes were produced for PMS interiors with a wide range of masses and rotations for comparison

\footnotetext{
${ }^{14}$ After this paper was submitted, a closely related study by Flaccomio et al. (2002) was released. It is based on the detection of 342 out of 696 unabsorbed ONC stars with the Chandra High Resolution Camera, which, unlike ACIS, does not give spectral information on the sources. Many of their findings are similar to ours: no correlation of $\log L_{t} / L_{\mathrm{bol}}$ with rotational period, strong correlation between $\log L_{t}$ and mass with lowluminosity outliers at intermediate masses; and a decline of $\log L_{t}$ with stellar age. One difference concerns the X-ray relationship to circumstellar disks: using the strength of the $\mathrm{Ca}$ II triplet lines as an indicator of accretion (in contrast to our photometric and imaging disk indicators), they find that low-accretion ONC stars have an order of magnitude higher X-ray luminosity than high-accretion stars. This is further evidence that young stellar $\mathrm{X}$-rays are not primarily produced in disk fields.
}

with our findings. Initial models of this type have been reported by Kitchatinov (2001), Küker \& Stix (2001) and references therein.

Despite these difficulties, the results seem to favor certain interpretations. The absence of an activity-rotation relation is by itself a good argument for some form of distributed dynamo arising throughout the convective zone, rather than the standard $\alpha-\Omega$ dynamo involving a tachocline. The change of X-ray properties at intermediate masses when a radiative core appears, and the scaling between X-ray emission and the volume of the convective region at lower masses, together lend support for a distributed dynamo interpretation for T Tauri stars.

However, we cannot yet exclude alternatives such as a standard dynamo in a "saturated" or "supersaturated" regime, where the saturation level occurs at a substantially lower value of $L_{t} / L_{\mathrm{bol}}$ than in main-sequence stars. If PMS stars indeed all have "saturated" dynamos, it is possible that little will be learned of their magnetic processes, especially as we do not understand the cause of saturation even in main-sequence stars. Similarly, magnetic reconnection of a mass-dependent fossil field may still be a viable model. However, the findings do not support models in which the $\mathrm{X}$-rays are associated with a circumstellar disk, either reconnection of star-disk fields at the corotation radius or reconnection of sheared disk-disk fields.

Additional forthcoming X-ray observations on the ONC should provide critical new insights. A 10 day Chandra ACIS observation is planned which will give an order of magnitude increase in sensitivity, essential for tracing magnetic activity in $M \leq 0.7 M_{\odot}$ PMS stars, and a sufficiently long time series of all stars to obtain detailed characteristics of PMS X-ray emission and variability. Several relevant studies are planned. The statistical properties of X-ray flares (e.g., the distribution of energies, durations, and recurrence rates) may reveal similarities or differences when compared to flares in the Sun and older active stars. Quiescent X-ray levels between flares will be sought, and may show less scatter in correlations with other stellar properties than we find here. We will search for rotationally modulated X-ray-emitting structures that might reveal large-scale asymmetries in the magnetic field geometry predicted by $\alpha-\alpha$ dynamos and relic core fields. Conceivably, transitions in the strength and structure of reconnecting surface magnetic fields reflecting the emergence of a core radiative zone will be seen in comparisons of younger versus older and less versus more massive PMS stars.

We are very appreciative of the careful and insightful reading of the manuscript by Dermott Mullan (Bartol) and the anonymous referee. E. D. F. also greatly benefited from discussions with participants of stellar magnetism workshops in Santiago, Boulder, and Toulouse during 20012002. Patrick Broos (Penn State), Steven Pravdo (JPL), and Yohko Tsuboi (Penn State/Chuo) played critical roles in the Chandra ACIS Orion project. Sofia Randich (Arcetri) and William Herbst (Wesleyan) provided valuable unpublished results and comments. This work was principally supported by NASA contract NAS 8-38252 (Garmire, PI). 
REFERENCES

Alcalá, J. M., Covino, E., Torres, G., Sterzik, M. F., Pfeiffer, M. J. \& Neuhäuser, R. 2000, A\&A, 353, 186

Ayres, T. R., Linsky, J. L., Vaiana, G. S., Golub, L., \& Rosner, R. 1981, ApJ, 250, 293

Barnes, S., Sofia, S., \& Pinsonneault, M. 2001, ApJ, 548, 1071

Birk, G. T., Schwab, D., Wiechen, H., \& Lesch, H. 2000, A\&A, 358, 1027

Bodenheimer, P. 1995, ARA\&A, 33, 199

Bouvier, J. 1990, AJ, 99, 946

Bouvier, J., Bertout, C., Benz, W., \& Mayor, M. 1986, A\&A, 165, 110

Bouvier, J., Forestini, M., \& Allain, S. 1997a, A\&A, 326, 1023

Bouvier, J., et al. 1997b, A\&A, 318, 495

Brun, A. S. 2002, in Magnetism and Activity of the Sun and Stars, ed. J. Arnaud (Les Ulis: EAS), in press

Carpenter, J. M., Hillenbrand, L. A., \& Skrutskie, M. F. 2001, AJ, 121, 3160

Casanova, S., Montmerle, T., Feigelson, E. D., \& Andre, P. 1995, ApJ, 439, 752

Charbonneau, P., Christensen-Dalsgaard, J., Henning, R., Larsen, R. M.,

Schou, J., Thompson, M. J., \& Tomczyk, S. 1999, ApJ, 527, 445

Charbonneau, P., \& MacGregor, K. B. 1997, ApJ, 486, 502

Damiani, F., \& Micela, G. 1995, ApJ, 446, 341

D’Antona, F., \& Mazzitelli, I. 1997, Mem. Soc. Astron. Italiana, 68, 807

D’Antona, F., Ventura, P., \& Mazzitelli, I. 2000, ApJ, 543, L77

Delfosse, X., Forveille, T., Perrier, C., \& Mayor, M. 1998, A\&A, 331, 581

Donati, J.-F. 1999, MNRAS, 302, 457

Dudorov, A. E., Krivodubskii, V. N., Ruzmaikina, T. V., \& Ruzmaikin, A. A. 1989, Soviet Astron., 33, 420

Durney, B. R., De Young, D. S., \& Roxburgh, I. W. 1993, Sol. Phys., 145, 207

Durney, B. R., \& Robinson, R. D. 1982, ApJ, 253, 290

Feigelson, E. D., Broos, P., Gaffney, J. A., Garmire, G., Hillenbrand, L. H., Pravdo, S. H., Townsley, L., \& Tsuboi, Y. 2002a, ApJ, 574, 258 (F02a)

Feigelson, E. D., Casanova, S., Montmerle, T., \& Guibert, J. 1993, ApJ, 416, 623

Feigelson, E. D., Garmire, G. P., \& Pravdo, S. H. 2002b, ApJ, 572, 335 (F02b)

Feigelson, E. D., \& Montmerle, T. 1999, ARA\&A, 37, 363

Flaccomio, E., Damiani, F., Micela, G., Sciortino, S., Harnden, F. R., Murray, S. S., \& Wolk, S. J. 2002, ApJ, 582, 398

Fleming, T. A., Schmitt, J. H. M. M., \& Giampapa, M. S. 1995, ApJ, 450, 401

Gagné, M., Caillault, J., \& Stauffer, J. R. 1995, ApJ, 445, 280

Gaidos, E. J. 1998, PASP, 110, 1259

Garmire, G., Feigelson, E. D., Broos, P., Hillenbrand, L. A., Pravdo, S. H., Townsley, L., \& Tsuboi, Y. 2000, AJ, 120, 1426

Getman, K. V., Feigelson, E. D., Townsley, L., Bally, J., Lada, C. J., \& Reipurth, B. 2002, ApJ, 575, 354

Gilliland, R. L. 1986, ApJ, 300, 339

Glassgold, A. E., Feigelson, E. D., \& Montmerle, T. 2000, in Protostars and Planets IV, ed. V. Mannings et al. (Tucson: Univ. Arizona Press), 429

Gondoin, P. 1999, A\&A, 352, 217

Granzer, T., Schüssler, M., Caligari, P., \& Strassmeier, K. G. 2000, A\&A, 355,1087

Güdel, M., Guinan, E. F., \& Skinner, S. L. 1997, ApJ, 483, 947

Hartmann, L. 1998, Accretion Processes in Star Formation (New York: Cambridge Univ. Press)

2001, AJ, 121, 1030

Herbst, W., Rhode, K. L., Hillenbrand, L. A., \& Curran, G. 2000, AJ, 119, 261

Herbst, W., et al. 2002, ApJ, submitted

Hillenbrand, L. A 1997, AJ, 113,1733

Hillenbrand, L. A., \& Carpenter, J. M. 2000, ApJ, 540, 236

Hillenbrand, L. A., Strom, S. E., Calvet, N., Merrill, K. M., Gatley, I., Makidon, R. B., Meyer, M. R., \& Skrutskie, M. F. 1998, AJ, 116, 1816

Houdebine, E. R., \& Stempels, H. C. 1997, A\&A, 326, 1143

Huensch, M., Schmitt, J. H. M. M., Schroeder, K.-P., \& Reimers, D. 1996, A\&A, 310, 801

Ihaka, R., \& Gentleman, R. 1996, J. Comput. Graphical Stat., 5, 299

Imanishi, K., Koyama, K., \& Tsuboi, Y. 2001, ApJ, 557, 747

James, D. J., Jardine, M. M., Jeffries, R. D., Randich, S., Collier Cameron, A., \& Ferreira, M. 2000, MNRAS, 318, 1217

Jardine, M., \& Unruh, Y. C. 1999, A\&A, 346, 883

Johns-Krull, C. M., Valenti, J. A., \& Koresko, C. 1999, ApJ, 516, 900

Jones, B. F. \& Walker, M. F. 1988, AJ 95, 1755

Kastner, J. H., Zuckerman, B., Weintraub, D. A., \& Forveille, T. 1997, Science, 277, 67

Kim, Y., \& Demarque, P. 1996, ApJ, 457, 340

Kitchatinov, L. L. 2001, Astron. Rep., 45, 816
Kitchatinov, L. L., Jardine, M., \& Collier Cameron, A. 2001, A\&A, 374, 250

Kitchatinov, L. L., \& Rüdiger, G. 1999, A\&A, 344, 911

König, B., Neuhäuser, R., \& Stelzer, B. 2001, A\&A, 369, 971

Koyama, K., Hamaguchi, K., Ueno, S., Kobayashi, N., \& Feigelson, E. D. 1996, PASJ, 48, L87

Krishnamurthi, A., et al. 1998, ApJ, 493, 914

Küker, M., \& Rüdiger, G. 1997, A\&A, 328, 253 1999, A\&A, 346, 922

Küker, M., \& Stix, M. 2001, A\&A, 366, 668

Lawson, W. A., Crause, L. A., Mamajek, E. E., \& Feigelson, E. D. 2001, MNRAS, 321, 57

Levy, E. H., \& Araki, S. 1989, Icarus, 81, 74

Levy, E. H., Ruzmaikin, A. A., \& Ruzmaikina, T. V. 1991, in The Sun in Time, ed. C. Sonett et al. (Tucson: Univ. Arizona Press), 589

Luhman, K. L. 2000, ApJ, 544, 1044

Mamajek, E. E., Lawson, W. A., \& Feigelson, E. D. 2000, ApJ, 544, 356

Markiel, J. A., \& Thomas, J. H. 1999, ApJ, 523, 827

Mathieu, R. D. 1994, ARA\&A, 32, 465

McGill, R., Tukey, J. W., \& Larsen, W. A. 1978, Am. Statistician, 32, 12

Merloni, A., \& Fabian, A. C. 2001, MNRAS, 328, 958

Mestel, L. 1999, Stellar Magnetism (Oxford: Clarendon)

Montesinos, B., Thomas, J. H., Ventura, P., \& Mazzitelli, I. 2001 MNRAS, 326, 877

Montmerle, T., \& Grosso, N. 2002, in The Origin of Stars and Planets, ed. J. Alves (Garching: ESO), 453

Montmerle, T., Grosso, N., Tsuboi, Y., \& Koyama, K. 2000, ApJ, 532 1097

Montmerle, T., Koch-Miramond, L., Falgarone, E., \& Grindlay, J. E. 1983, ApJ, 269, 182

Moss, D. 1996, A\&A, 305, 140

2002, in Magnetism and Activity of the Sun and Stars, ed. J. Arnaud (Les Ulis: EAS), in press

Mullan, D. J. 1973, Irish Astron. J., 11, 32

1984, ApJ, 282, 603

Mullan, D. J., \& MacDonald, J. 2001, ApJ, 559, 353

Neuhäuser, R., Sterzik, M. F., Schmitt, J. H. M. M., Wichmann, R., \& Krautter, J. 1995, A\&A, 297, 391

Noyes, R. W., Hartmann, L. W., Baliunas, S. L., Duncan, D. K., \& Vaughan, A. H. 1984, ApJ, 279, 763

O’Dell, C. R. 2001, ARA\&A, 39, 99

Palla, F. 2001, in ASP Conf. Ser. 243, From Darkness to Light: Origin and Evolution of Young Stellar Clusters, ed. T. Montmerle \& Ph. André (San Francisco: ASP), 525

Palla, F., \& Stahler, S. W. 1993, ApJ, 418, 414

Pallavicini, R., Golub, L., Rosner, R., Vaiana, G. S., Ayres, T., \& Linsky, J. L. 1981, ApJ, 248, 279

Parker, E. N. 1993, ApJ, 408, 707

Pizzolato, N., Maggio, A., \& Sciortino, S. 2000, A\&A, 361, 614

Preibisch, T. 1997, A\&A, 320, 525

Preibisch, T., \& Zinnecker, H. 2002, AJ, 123, 1613

Randich, S. 1998, in ASP Conf. Ser. 154, Cool Stars, Stellar Systems and the Sun, ed. R. A. Donahue \& J. A. Bookbinder (San Francisco: ASP), 501

. 2000, in ASP Conf. Ser. 198, Stellar Clusters and Associations Convection, Rotation and Dynamos, ed. R. Pallavicini et al. (San Francisco: ASP), 401

Randich, S., Schmitt, J. H. M. M., Prosser, C. F., \& Stauffer, J. R. 1996 A\&A, 305, 785

Rhode, K. L., Herbst, W., \& Mathieu, R. D. 2001, AJ, 122, 3258

Romanova, M. M., Ustyugova, G. V., Koldoba, A. V., Chechetkin, V. M., \& Lovelace, R. V. E. 1998, ApJ, 500, 703

Rosner, R., Musielak, Z. E., Cattaneo, F., Moore, R. L., \& Suess, S. T. 1995, ApJ, 442, L25

Schrijver, C. J., \& Zwaan, C. 2000, Solar and Stellar Magnetic Activity (Cambridge: Univ. Cambridge Press)

Shu, F. H., Shang, H., Glassgold, A. E., \& Lee, T. 1997, Science, 277, 1475

Siess, L. 2001, in ASP Conf. Ser. 243, From Darkness to Light: Origin and Evolution of Young Stellar Clusters, ed. T. Montmerle \& Ph. André (San Francisco: ASP), 581

Sills, A., Pinsonneault, M. H., \& Terndrup, D. M. 2000, ApJ, 534, 335

Stassun, K. G., Mathieu, R. D., Mazeh, T., \& Vrba, F. J. 1999, AJ, 117, 2941

Stelzer, B., \& Neuhäuser, R. 2001, A\&A, 377, 538

Stepień, K. 2000, A\&A, 353, 227

Stepień, K., Schmitt, J. H. M. M., \& Voges, W. 2001, A\&A, 370, 157

Strassmeier, K. G., Fekel, F. C., Gray, D. F., Hatzes, A. P., Schmitt, J. H. M. M., \& Solanki, S. K. 1998, in ASP Conf. Ser. 154, Cool Stars, Stellar Systems and the Sun, ed. R. A. Donahue \& J. A. Bookbinder (San Francisco: ASP), 257

Tayler, R. J. 1987, MNRAS, 227, 553

Thelen, J.-C., \& Cattaneo, F. 2000, MNRAS, 315, L13

Tinker, J., Pinsonneault, M., \& Terndrup, D. 2002, ApJ, 564, 877 
Tukey, J. W. 1977, Exploratory Data Analysis (Reading: Addison-Wesley) Vilhu, O., \& Walter, F. M. 1987, ApJ, 321, 958

Walter, F. M., \& Boyd, W. T. 1991, ApJ, 370, 318

Walter, F. M., \& Kuhi, L. V. 1981, ApJ, 250, 254
Weisskopf, M. C., Brinkman, B., Canizares, C., Garmire, G., Murray, S., \& Van Speybroeck, L. P. 2002, PASP, 114, 1

Wichmann, R et al. 2000, A\&A, 359, 181

Wuchterl, G., \& Klessen, R. S. 2001, ApJ, 560, L185 